\title{
Practical Inductive Link Design for Biomedical Wireless Power Transfer: A Tutorial
}

\author{
Matthew Schormans (1), Student Member, IEEE, Virgilio Valente (i), Member, IEEE, \\ and Andreas Demosthenous ${ }^{(0)}$, Fellow, IEEE
}

\begin{abstract}
Wireless power transfer systems, particularly those based on inductive coupling, provide an increasingly attractive method to safely deliver power to biomedical implants. Although there exists a large body of literature describing the design of inductive links, it generally focuses on single aspects of the design process. There is a variety of approaches, some analytic, some numerical, each with benefits and drawbacks. As a result, undertaking a link design can be a difficult task, particularly for a newcomer to the subject. This tutorial paper reviews and collects the methods and equations that are required to design an inductive link for biomedical wireless power transfer, with a focus on practicality. It introduces and explains the published methods and principles relevant to all aspects of inductive link design, such that no specific prior knowledge of inductive link design is required. These methods are also combined into a software package (the Coupled Coil Configurator), to further simplify the design process. This software is demonstrated with a design example, to serve as a practical illustration.
\end{abstract}

Index Terms-Implantable devices, inductive coupling, inductive links, wireless power transfer.

\section{INTRODUCTION}

W IRELESS power transfer (WPT) is a field that is rapidly growing and is relevant across a range of domains, including industrial applications [1]-[3], consumer products [4]-[6], and biomedical devices [7]-[13]. In the context of biomedical implants and devices, WPT has been instrumental in progressing the state of the art. The first fully implantable device was Greatbatch's pacemaker circa 1958 [14], which required the use of mercury based batteries; such a device would be considered unsafe for implantation today. While many modern implants still employ batteries, implants that

Manuscript received March 18, 2018; revised May 16, 2018; accepted June 4, 2018. Date of publication July 13, 2018; date of current version October 19,2018 . This work was supported by a Ph.D. studentship awarded to M. Schormans by the Engineering and Physical Sciences Research Council under Grant EP/M506448/1. This paper was recommended by Associate Editor M. Sawan. (Corresponding author: Andreas Demosthenous.)

M. Schormans and A. Demosthenous are with the Department of Electronic and Electrical Engineering, University College London, London WC1E 7JE, U.K. (e-mail: matthew.schormans.10@ucl.ac.uk; a.demosthenous@ucl.ac.uk).

V. Valente was with the Department of Electronic and Electrical Engineering, University College London, London WC1E 7JE, U.K. He is now with the Department of Microelectronics, Delft University of Technology, 2628, CD Delft, The Netherlands (e-mail: v.valente@tudelft.nl).

Color versions of one or more of the figures in this paper are available online at http://ieeexplore.ieee.org.

Digital Object Identifier 10.1109/TBCAS.2018.2846020
TABLE I

COMPARISON OF DIFFERENT WORK EMPLOYING INDUCTIVE LINKS FOR BIOMEDICAL DEVICES

\begin{tabular}{|c|c|c|c|c|c|}
\hline Work & Year & Power & Eff. & Freq. & Application Notes \\
\hline [23] & 2010 & $11.2 \mathrm{~mW}$ & $14 \%$ & $\begin{array}{l}13.56 \\
\mathrm{MHz}\end{array}$ & $\begin{array}{c}\text { Low power implant } \\
\text { powering with CotS } \\
\text { devices. }\end{array}$ \\
\hline [26] & 2015 & $30 \mathrm{~W}$ & $95 \%$ & $\begin{array}{l}800 \\
\mathrm{kHz}\end{array}$ & $\begin{array}{l}\text { Implanted ventricular } \\
\text { assist device powering. }\end{array}$ \\
\hline [25] & 2017 & $100 \mathrm{~mW}$ & $60 \%$ & $\begin{array}{l}13.56 \\
\mathrm{MHz}\end{array}$ & $\begin{array}{l}\text { Simultaneous power and } \\
\text { data transfer for } \\
\text { implants. }\end{array}$ \\
\hline [27] & 2017 & $1.3 \mathrm{~mW}$ & $2.4 \%$ & $\begin{array}{l}60 \\
\mathrm{MHz}\end{array}$ & $\begin{array}{l}\text { Power transfer to many } \\
\text { distributed } \\
\text { micro-implants. }\end{array}$ \\
\hline$[28]$ & 2018 & $9.2 \mathrm{~mW}$ & $75 \%$ & $\begin{array}{l}13.56 \\
\mathrm{MHz}\end{array}$ & $\begin{array}{c}\text { Single-chip } \\
\text { simultaneous power and } \\
\text { data transfer for } \\
\text { implants. }\end{array}$ \\
\hline
\end{tabular}

employ WPT are smaller, and do not require explantation for battery recharging [15], [16].

The wide range of today's implantable devices, including pacemakers, cochlear implants, and neurostimulators, adopt a similarly wide variety of WPT methods, including inductively coupled power transfer (ICPT) [7]-[13], ultrasonic power transfer (UPT) [17], [18], and capacitively coupled power transfer (CCPT) [19]-[21]. Of these methods, ICPT can be considered the oldest and most established for biomedical WPT. For example, an electrocardiogram system employing ICPT was presented by Ko et al. in 1979 [22].

ICPT is used in a variety of state of the art implants, since it can efficiently transfer power over short distances, and can be scaled over a range of output power levels, from milliwatts to tens of watts [23]-[28]. Particularly in the case of high power delivery, the total system efficiency can be $>95 \%$ [26]. Some examples of state-of-the-art biomedical ICPT systems are summarized in Table I.

This combination of scalability between low and high power devices and high potential system efficiency makes ICPT attractive compared to other methods such as UPT or CCPT. Despite the flexibility, prevalence, and potential of ICPT, there is still no firm consensus regarding methods of inductive link design and optimization. Terman provides some of the earliest analysis of inductively coupled systems [29], [30]. However, Terman's analysis alone is insufficient to fully design an inductive link; factors such as efficiency and resilience must also be 
considered, where Terman's analysis focuses on gain and bandwidth. There has been a lot of subsequent analysis dedicated to characterizing and optimizing such links, however these analyses have shortcomings. For instance, they are often very focused on a specific aspect or design procedure, and so only provide insight into a small part of the design process [31]-[35]. Donaldson and Perkins [33] give a thorough analysis of the effects of varying coupling coefficients on link performance of seriesparallel links; however, no other link configurations are considered. Jow and Ghovanloo [31] consider a detailed model of PCB based spiral coils, but the analysis is not immediately applicable to wirewound coils. Alternately, some analyses cover a wider range of aspects in great detail [36]-[38], but are often impractical. For instance Halpern and Ng [36] give a very detailed analysis of the limits of power delivery and efficiency of two-coil series-series and series-parallel configured links, but significant prior knowledge of the subject is required to apply it to a design process.

In addition, while these methods provide a good picture of link characteristics in the theoretical circuit domain, they assume the link parameters are known a priori. The problem with this assumption is that the electrical parameters of the coils must be determined through some means beforehand, generally through fabrication and measurement or finite element method (FEM) simulation [3], [39]-[41]. FEM simulations can allow link behavior to be predicted more accurately than calculations alone. However, FEM software is often expensive, with a steep learning curve. Although fabrication and measurement is cheap, requiring measurements as part of the design procedure is time consuming and impractical. FEM can also be equally time consuming over many iterations.

The full design process therefore requires more than just consideration of the electrical parameters of the link. There are inevitably design restrictions on elements such as coil size, materials, and separations/displacements. As a result, it is imperative to be able to easily convert between geometric and electrical parameters as part of an analytic design process. In addition, it is beneficial to consider a broader approach than those presented in older analyses (e.g. [29], [30], [42]), where assumptions are made to simplify the calculations, and parameters such as the frequency, coupling, losses, and load are considered constant [42].

The overall goal of this paper is to provide an approachable introduction to the subject of 2-coil inductive link design. This goal can be broken down into three main aims:

1) To provide a sequence of analytic equations describing coil parameters and link behaviour, that can be used to form a design process.

2) To present these methods, starting from first principles, to give newcomers to the subject an introduction to the design process, and key compromises and tradeoffs to consider.

3) To provide a software tool based on the presented methods, to facilitate the design process and make understanding link behaviour more intuitive.

The methods presented here are the result of the collection, review, and selection of the most relevant design approaches

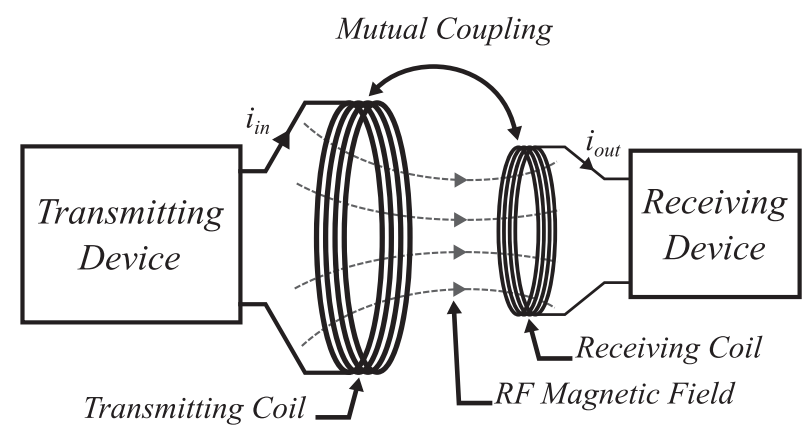

Fig. 1. Conceptual overview of ICPT.

present in the literature; they are considered both sufficiently practical and accurate for the designing and prototyping process. In addition to specific methodology, the paper provides useful rules for the designer to follow, to give a sense of how to apply theory to practice. The focus is on biomedical applications, but the principles can easily be applied to design inductive links for other purposes. The accompanying free software tool ' $\mathrm{CuCCo}$ ' [43], was written with the aim of giving the designer a set of easy to use scripts that perform all the necessary calculations required for designing an inductive link.

The paper is structured as follows. Section II reviews the basic theory of operation for ICPT and reviews the essential principles. Section III considers the problem of link design in detail, and presents analytical methods for geometric to electrical parameter conversion, as well as link characterization. Section IV discusses important practical considerations and tradeoffs that are inherent to link design. Finally, Section V gives a design example, demonstrating the methods presented and usage of CuCCo.

\section{PRINCIPLES OF ICPT}

Any WPT system that uses near-field coupled inductive coils can be considered as some form of ICPT. Even though a wide variety of designs exists, all ICPT systems employ at least two coils that are closely coupled relative to the coil diameters. Although some designs use 3 or 4 coils [1], [3], [27], [44], [45], the analysis in this paper is limited to the 2-coil case, as it is the simplest and most practical ICPT arrangement.

In the simplest case, ICPT can be achieved by aligning two inductive coils in close proximity, such that they are magnetically coupled. This principle is illustrated in Fig. 1, where energy is transferred from the transmitting coil (Tx) to the receiving coil $(\mathrm{Rx})$ due to the magnetic field of the Tx being coupled into the Rx. This can be considered as a pair of inductances $L_{1}$ and $L_{2}$, coupled by a mutual inductance $M$. Assuming that parasitic capacitance in the coils is negligible, this forms a nonresonant system. Non-resonant energy transfer is not generally used for WPT, as its efficiency is poor compared to resonant arrangements [42].

By adding capacitors in series or parallel with the coils resonant arrangements can be realized, referred to as near field resonant inductive coupling (NRIC) [16]. NRIC is a form of 


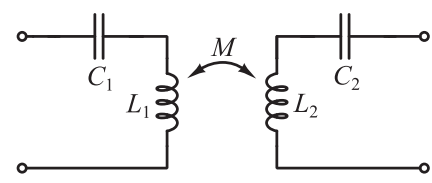

(a)

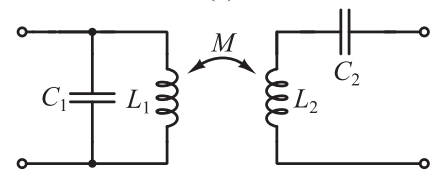

(c)

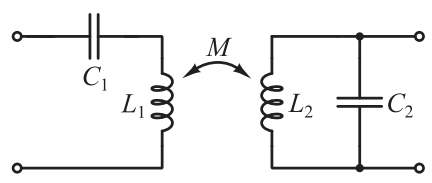

(b)

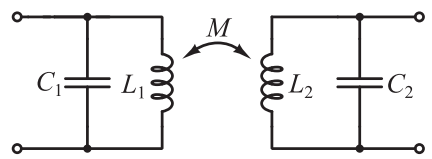

(d)
Fig. 2. Comparison of NRIC link arrangements. (a) Series-Series (SS), (b) Series-Parallel (SP), (c) Parallel-Series (PS), (d) Parallel-Parallel (PP).

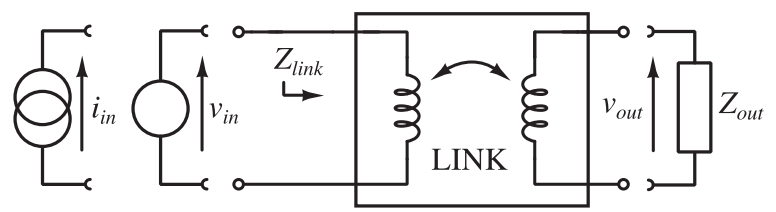

Fig. 3. General 2-port model. An appropriate source should be chosen depending on resonant configuration.

ICPT that can achieve increased efficiency compared to the non-resonant case. Fig. 2 shows the four possible arrangements for two-coil NRIC; each coil has an accompanying capacitor, so each side of the link forms a resonant tank. Each of the configurations in Fig. 2 can be considered as a 2-port network, where the $\operatorname{Tx}\left(L_{1}\right)$ is driven by a power source and the $\operatorname{Rx}\left(L_{2}\right)$ is connected to a load. The 2-port representation is illustrated in Fig. 3, where $Z_{\text {link }}$ is the impedance looking into the link, and will depend on the topology chosen from Fig. 2. $Z_{\text {out }}$ is the impedance connected to the output port of the link. For good input matching, a series resonant primary (Fig. 2(a,b)) is driven by a voltage source, and a parallel resonant primary (Fig. 2(c,d)) is driven by a current source.

Using the circuit analysis presented by Terman [29], [30], parameters such as the link gain and impedance can be determined for any of the link configurations in Fig. 2. The gain is considered either as a unitless voltage gain $\left(v_{\text {out }} / v_{\text {in }}\right)$, or as a transimpedance $\left(v_{\text {out }} / i_{\text {in }}\right)$, depending on the driver. This analysis is developed further by Van Schuylenbergh and Puers, where equations for link efficiency and optimization procedures are developed and presented [42]. Combining these analyses is sufficient to perform rudimentary analysis of an inductive link in terms of the electrical circuit parameters, extracting parameters such as the power delivered to the load (PDL) and power transfer efficiency (PTE). This forms the basis of the electrical parameter analyses in Section III.

\section{LINK DESIGN}

This section summarizes the key analyses required for link design. By using the analyses in this section, it is possible to design links to achieve design targets for a given application. Fig. 4 is an overview of a typical design flow for designing an inductive link. The process starts with constraints and targets. Constraints typically include geometric limits, e.g. the receiver coil size must fit within an implant's package, and targets describe the desired performance metrics. Examples include PTE, PDL, and tolerance to displacement and load variations. The process then involves determining geometry that fits the initial constraints, extracting the electrical parameters associated with that geometry, using these extracted parameters to predict the link performance, and then comparing that performance with the design targets. This process is then repeated, altering the geometry as necessary until the predicted performance is acceptable. The designer must tradeoff between small coils, PTE/PDL, and displacement tolerance; see the triad of features inset at the top of Fig. 4. For example, to obtain good performance in PTE/PDL with small coils, the displacement tolerance will suffer, i.e. the link will only achieve the desired performance for a small range of displacement values. The rest of this section collects relevant design equations from the literature, and describes how they can be used according to the flow presented in Fig. 4. This can be considered in two parts: coil modelling, where electrical parameters are extracted from the proposed geometry; and link modelling, where the performance of the link is predicted in terms of these extracted electrical parameters.

\section{A. Coil Modelling}

While any coil types can be used for WPT, in general the coil types used are short wire solenoids, printed circuit board (PCB) spirals, or 'pancake' coils. Fig. 5 shows the structure of solenoid and PCB spiral coils, with their geometric parameters annotated. Pancake coils can be considered analogous to PCB spiral coils, but would use the $d_{0}$ and $p$ parameters for wires, rather than the $w$ and $s$ parameters for PCB tracks. These geometric parameters must then be converted to electrical parameters: inductance, resistance, and capacitance, shown in Fig. 6; a full list of parameters is given in Table II.

1) Inductance: The procedure of analytic inductance calculation is well described in the literature. Wheeler [46] provides one of the earliest expressions for inductance calculation, where simple formulae are given for calculating the inductance of long radio coils. A development of this work is given by Grover [47], where a collection of tables and methods are presented; again the focus is on radio coil design. The subject has subsequently been reformulated and developed, generally building upon the work of Grover and Wheeler [48]-[50]. Hurley et al. in particular provide analysis of coil parameters in the context of WPT [49]. Perhaps the most thorough treatment of the subject to date however is attributable to Knight, where the inductor is considered from first principles, and the appropriate theory is developed in order to produce accurate expressions for calculating inductance [51]. Knight provides a continuous expression for calculating the inductance of solenoid coils of any proportion, based on the current-sheet inductance method:

$$
L=\frac{\mu_{0} \pi r^{2} n^{2} \kappa}{l}
$$




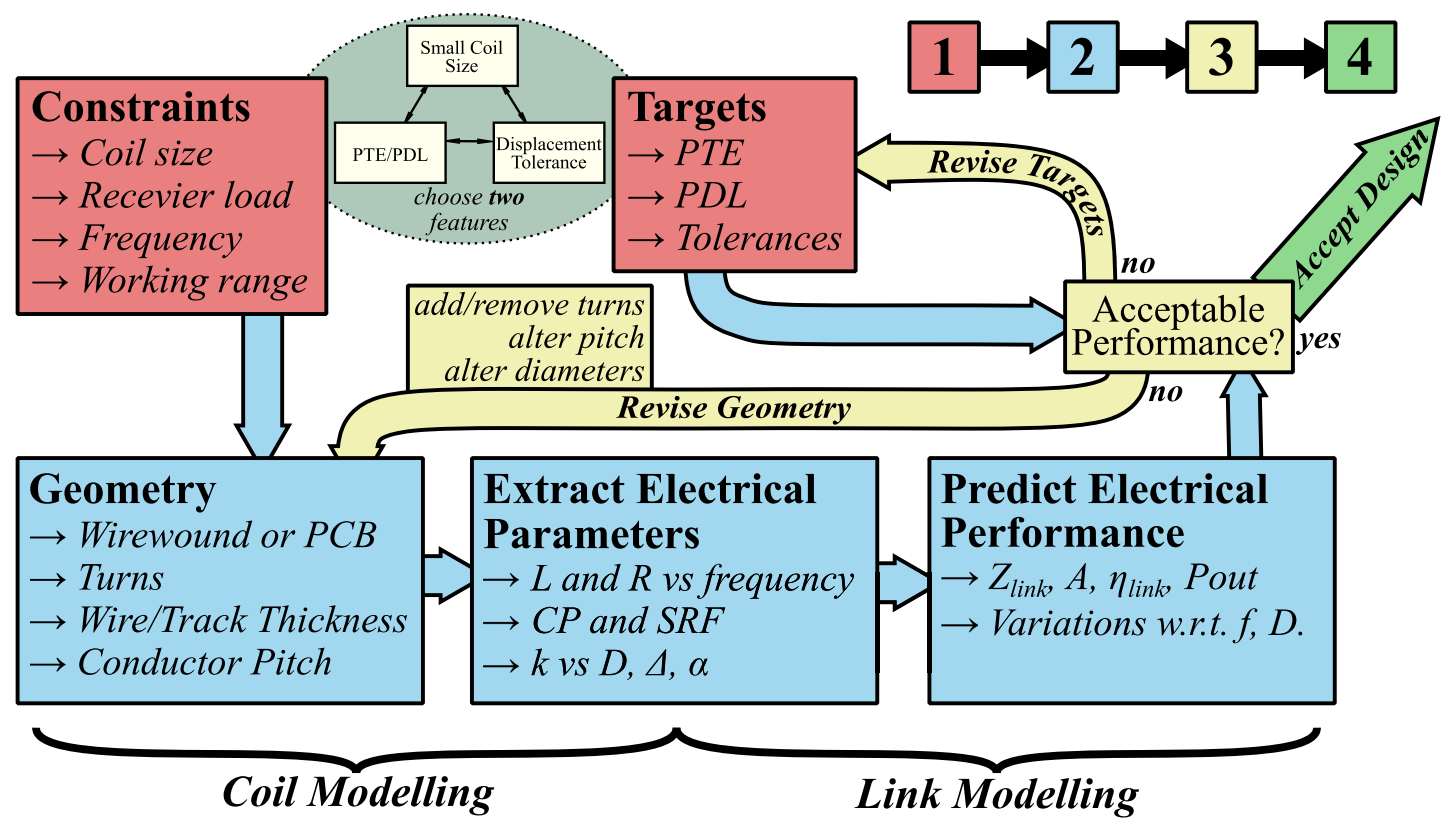

Fig. 4. General flow diagram for the initial design of inductive links.

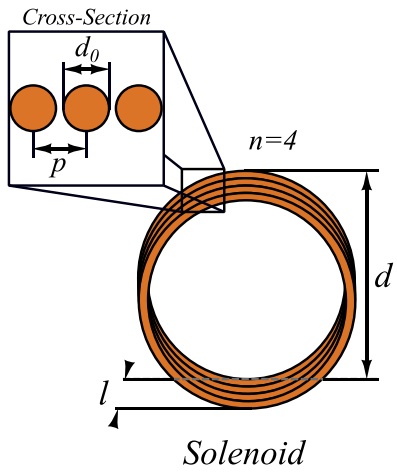

Fig. 5. Solenoid and spiral structures. Geometric parameters are annotated.

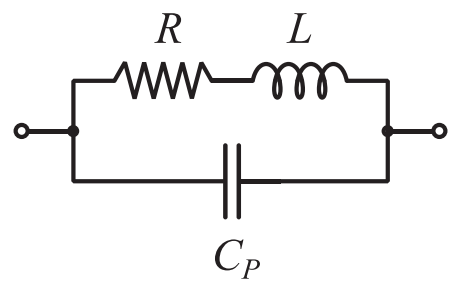

Fig. 6. Solenoid and spiral structures. Geometric parameters are annotated.

where $\mu_{0}$ is the permeability of free space, $r=d / 2$, and $\kappa$ is Nagaoka's coefficient, approximated as [51]:

$$
\kappa=z_{k}\left(\ln \left(1+\frac{1}{z_{k}}\right)+\frac{1}{\left(k_{0}+k_{1}\left(\frac{l}{d}\right)^{2}+\frac{w_{1}}{\left(\left|w_{2}\right|^{2}+d / l\right)^{v}}\right)}\right)
$$

TABLE II

COIL PARAMETERS

\begin{tabular}{cc|cc}
\hline & Geometric & \multicolumn{2}{c}{ Electrical $^{\dagger}$} \\
\hline$d$ & Diameter & $L$ & Inductance \\
$l$ & Length & $R$ & Series Loss \\
$n$ & Number of Turns & $Q$ & Quality Factor \\
$d_{0}$ & Wire Diameter & $C$ & Resonating Capacitance \\
$p$ & Winding Pitch & $C_{P}$ & Parasitic Capacitance \\
$w$ & Track Width & - & - \\
$s$ & Track Spacing & - & - \\
\hline
\end{tabular}

${ }^{\mp}$ These units are with reference to either the Tx or Rx coil, and are noted with subscript 1 or 2 respectively.

where $z_{k}=l /(\pi r), k_{0}=2.30038, k_{1}=3.437, k_{2}=1.76356$, $w_{1}=-0.47, w_{2}=0.755$, and $v=1.44$. By substituting this approximation for $\kappa$ into (1), $L$ can be calculated to within $\pm 20 \mathrm{ppM}$. While it is possible to evaluate the appropriate line integral to obtain an exact result [48], the procedure is complex and can be computationally expensive over many design iterations. Equations (1) and (2) however are simple expressions that are both computationally efficient and practical. It should be noted that the inductance value produced by (1) for a given geometry is valid only at low frequencies with respect to the self-resonant frequency (SRF) of the inductor. Determining the SRF is a complex topic itself, and is discussed further in Section III-A3.

For the case of PCB spiral coils, the inductance calculation is somewhat simpler. Mohan et al. give an expression based on the fill-factor $\beta=\left(d-d_{\text {in }}\right) /\left(d+d_{\text {in }}\right)$, and the average turn diameter $d_{\text {avg }}=0.5\left(d+d_{\text {in }}\right)$ [52]:

$$
L_{\mathrm{spiral}}=\frac{\mu n^{2} d_{\mathrm{avg}}}{2}\left(\ln \left(\frac{2.46}{\beta}\right)+0.2 \beta^{2}\right) .
$$




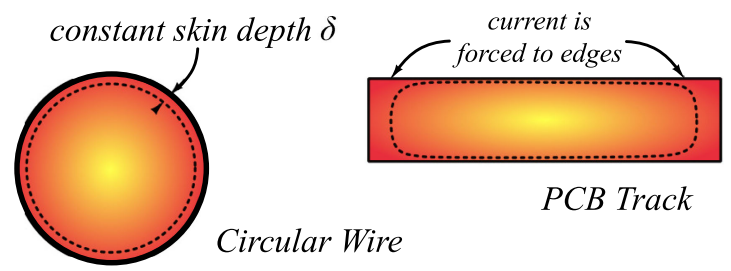

Fig. 7. Skin effect manifestation in wire and PCB trace.

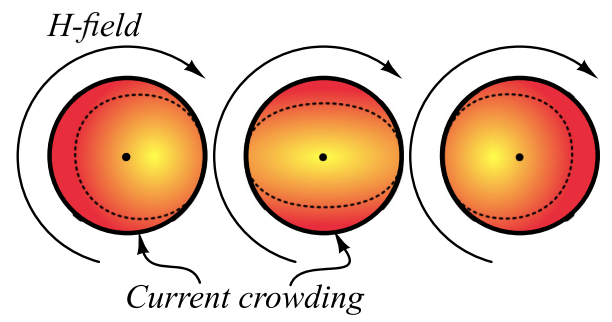

Fig. 8. Proximity effect causing uneven current crowding in adjacent conductors.

Equation (3) accurately predicts the inductance of a circular PCB spiral coil for most geometries (less than $8 \%$ error for $s \leq 3 w$ [52]). The expression in (3) can be modified for use with square, octagonal, and hexagonal coils, by adjusting the appropriate coefficients. This is detailed in Appendix B.

2) Series Loss: Determining the series loss R involves breaking down the loss into its constituent parts:

$$
R=R_{D C}+R_{\text {skin }}+R_{\text {prox }}
$$

where $R_{D C}$ is the simple resistive loss of the conductor, $R_{\text {skin }}$ is the loss due to the skin effect, and $R_{\text {prox }}$ is the loss due to the proximity effect. The skin effect is the formation of a thin 'skin' of current at the edges of a conductor that is carrying high frequency current. The effect is well documented; procedures to calculate $R_{\text {skin }}$ accurately for various conductor shapes appear as early as 1918 [53], [54]. Similar approaches have been used in recent years to predict skin effect losses in PCB spiral coils [31], [55], and wirewound coils [44] for biomedical WPT designs. Fig. 7 illustrates the manifestation of the skin effect in conductors of different cross section. In the case of the axially symmetric circular wire, current shaped by the skin effect can be considered to flow in a layer of continuous depth $\delta$. In the case of the PCB track, the distribution is less even, with current density being highest at the horizontal edges of the track.

To fully characterize the resistive losses however, the proximity effect must also be considered. The proximity effect describes the resistive loss that occurs due to adjacent current filaments flowing in the same direction. The closer the filaments, the greater the loss due to the proximity effect. Fig. 8 shows how the $\mathrm{H}$-field of one conductor interferes with the current density in adjacent conductors; the H-field generates an opposing current, which manifests as loss. Early work approximates the proximity effect losses as a constant proportion of the skin effect losses based on filament distance [48], [53]. Lammeraner and Stafl [56] consider the topic of both the skin and proximity effects in great detail from the perspective of Maxwell's equations, however the analysis is cumbersome and protracted. FEM based solutions are often used to predict proximity effect losses [57]-[60], or at least used to verify measurements [61]. More recently, the problem has been formulated into closed form expressions for the cases of round conductors; these solutions have been verified through both FEM simulation and measurements [62], [63]. The case of PCB spiral coils has also been considered, the proximity effect losses can be approximated using a similar process [55], [64]. Practical expressions for $R_{D C}, R_{\text {skin }}$, and $R_{\text {prox }}$ are given below. Firstly, the DC loss can be considered a function of the conductor's geometry and resistivity:

$$
R_{D C}=\rho \frac{l_{c}}{A_{c}},
$$

where $\rho$ is the resistivity of the material, $l_{c}$ is the length of the conductor, and $A_{c}$ is the cross-sectional area of the conductor. The length $l_{c}$ can be determined by:

$$
l_{c}=\pi d_{\mathrm{avg}} n
$$

where in the case of a single-layer solenoid $d_{\mathrm{avg}}=d$, and in the case of PCB spirals:

$$
d_{\mathrm{avg}}=d-\frac{(w+s) n}{2} .
$$

In the case of pancake wire spirals, the $(w+s)$ term can be replaced with the winding pitch $p$ to obtain the same result. $R_{\text {skin }}$ is simple to calculate in the case of a circular conductor; first, the skin depth $\delta$ can be defined as a function of frequency:

$$
\delta=\sqrt{\frac{2 \rho}{\omega \mu}}
$$

where $\mu \approx \mu_{0}$ for copper conductors. The loss due to the skin effect can therefore be considered in terms of the different cross sections of the full circular conductor and the current skin:

$$
R_{\text {skin }}=\frac{l_{c} \rho}{\pi\left(d_{0}-\delta\right) \delta} .
$$

For the case of a rectangular conductor, such as a PCB track, a convenient expression is given by Wheeler [65]:

$$
R_{\text {skin }}=R_{D C} \frac{t_{0}}{\delta\left(1-e^{-\frac{t_{0}}{\delta}}\right)} \frac{1}{1+\frac{t_{0}}{w}}
$$

where $t_{0}$ is the thickness of the PCB track (usually $\approx 35 \mu \mathrm{m}$ for standard plated PCBs). An expression for $R_{\text {prox }}$ is given by Kim and Park [63], which is applicable to any single-layer wire solenoid of otherwise arbitrary dimensions:

$$
R_{\text {prox }}=\frac{2 P_{\text {prox }}}{I_{0}^{2}} \approx 2 R_{D C} \pi^{2} r_{0}^{2}\left(\frac{2 r_{0}}{\delta}-1\right) \frac{H^{2}}{I_{0}^{2}}
$$

where $r_{0}=d_{0} / 2, P_{\text {prox }}$ is the power dissipation due to proximity effect losses, and $H$ is the cumulative $\mathrm{H}$-field experienced by the conductors in the solenoid. The analysis in [63] continues 


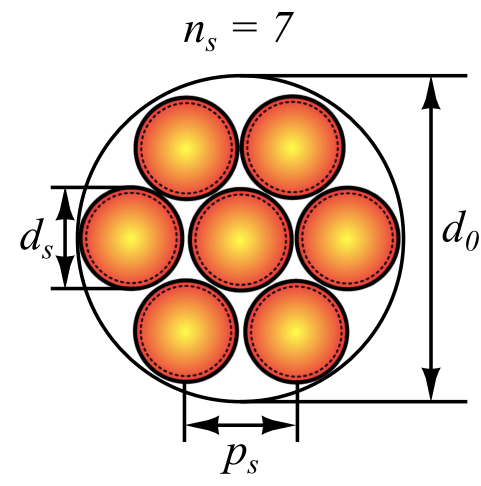

Fig. 9. A Litz wire bundle consisting of 7 strands, with strand diameter $d_{s}$ and strand pitch $p_{s}$.

by describing a process for determining the $\mathrm{H}$ - field experienced by each conductor, normalized against $I_{0}$ (the current flowing in the coil), and as a function of the relative distance to the other conductors in the coil. In this way the total H-field can be determined as a function of the geometry of the coil. The process of calculating the cumulative $\mathrm{H}$-field is detailed in Appendix B. In the case of PCB tracks, the significant asymmetry of the printed conductor cross-section (see Fig. 7) renders the approach in (11) inapplicable. An approximation by Kuhn and Ibrahim [64] defines the proximity effect loss in a printed spiral coil as:

$$
R_{\text {prox }}=\frac{R_{D C}}{10}\left(\frac{\omega}{\omega_{\text {crit }}}\right)^{2}
$$

where $\omega_{\text {crit }}$ is the critical frequency at which current crowding begins to occur:

$$
\omega_{\text {crit }}=\frac{3.1}{\mu_{0}} \frac{(w+s) \rho}{w^{2} t_{0}} .
$$

It is important to note that the approximation in (13) was initially specified for use with integrated coils; as a result it can lose accuracy at lower resisitivities. Utilizing (4)-(13) as appropriate, the losses due to both the skin and proximity effect can be accurately modelled in a simple manner, for the most common cases of single-layer solenoids and PCB spirals.

a) Litz Wire: Section III-A2 considers coils constructed of single core magnet wire (copper wire coated with a few microns of insulating polymer), or PCB traces. However, for lower frequency systems ( $<2 \mathrm{MHz}$ [30]) attempting to maximize Qfactor, it may be desirable to construct a coil from Litz wire [26], [66]-[69]. Litz wire consists of one or more bundles of thin magnet wire strands connected in parallel, primarily to reduce skin effect losses. By reducing skin effect losses in this way, Litz wire links can achieve greater efficiency than solid wire links that would fit in the same geometry, as long as the frequency can be kept low enough. A cross-section of a Litz wire bundle is shown in Fig. 9. Since each of the strands in a bundle is insulated from the others, each forms its own skin. As a result, a Litz coil will generally have a lower value of $R_{\text {skin }}$ than a solid wire coil with the same value of $d_{0}$ [70]. While Litz wire is a useful means of reducing skin effect losses, the proximity effect becomes a bigger issue, particularly at higher frequencies [71]. Modelling losses in Litz wire is a difficult task, and FEM techniques are commonly employed to simulate them [62], [72]. However, there are also some fairly simple approximate methods to predict losses in Litz wire coils [70], [73]. By simplifying the analysis of Bartoli et al. [70] to consider a Litz wire solenoid of only a single layer, the total $\mathrm{AC}$ loss $\left(R_{\text {skin }}+R_{\text {prox }}\right)$ can be approximated in terms of bessel functions as:

$$
\begin{aligned}
R_{A C} & =\pi d n R_{D C} n_{s} \frac{\gamma}{2}\left(A+2 \pi n_{s}\left(\zeta_{1}^{2}+\zeta_{2}^{2} \frac{\psi}{2 \pi n_{s}}\right) B\right), \\
A & =\frac{\operatorname{ber}(\gamma) b e i^{\prime}(\gamma)-b e i(\gamma) b e r^{\prime}(\gamma)}{\operatorname{ber}^{\prime 2}(\gamma)+b e i^{\prime 2}(\gamma)} \\
B & =\frac{\operatorname{ber}_{2}(\gamma) \operatorname{ber}^{\prime}(\gamma)-b e i_{2}(\gamma) b e i^{\prime}(\gamma)}{\operatorname{ber}^{2}(\gamma)+\operatorname{bei}^{2}(\gamma)}
\end{aligned}
$$

where $R_{D C}=\left(4 \rho l_{c}\right) /\left(n_{s} \pi d_{s}^{2}\right)$ is the DC loss, $\gamma=d_{s} /(\delta \sqrt{2})$, $\zeta_{1}=\left(d_{s} / p\right) \sqrt{\pi / 4}$ is an external porosity factor, $\zeta_{2}=$ $\left(d_{s} / p_{s}\right) \sqrt{\pi / 4}$ is an internal porosity factor ( $p_{s}$ is the average strand pitch (see Fig. 9)), and $\psi=\left(n \pi d_{s}^{2}\right) /\left(\pi d_{0}^{2}\right)$ is a packing factor. Equation (14) gives a good approximation of the total losses, as long as $\gamma \lesssim 1.2$ [70].

In all these cases, the calculated loss can be considered as a proportion of the coil's reactive impedance, in the form of its Q-factor:

$$
Q=\frac{\omega L}{R} .
$$

An ideal coil would have zero loss, i.e. $Q=\infty$. The $\mathrm{Q}$-factor is a useful parameter when considering PTE and PDL, and is discussed in Section III-B.

\section{3) Parasitic Capacitance}

As the drive frequency of an inductor is increased, it presents a changing impedance as it approaches its SRF, appearing as if a capacitor $C_{P}$ was connected in parallel. The source of this capacitance was originally considered to be the result of interwinding capacitance [74]. The basis of the theory is that due to each coil winding laying parallel to its neighbors, there exists a capacitance between them. The idea can be extended to include printed spiral coils by including additional capacitances seen between PCB layers as well as tracks [31], [55]. This principle has been used for the purposes of approximation [75], [76], but FEM has proved necessary for accuracy [77]. The basis of the inter-winding capacitance theory has been brought into contention however, with an alternate method that considers the coil as a transmission line being developed by Knight [51]. This analysis has been corroborated by Payne, who gives simple approximations for determining the SRF (in MHz), providing measured results accurate to within $0.2 \%$ of the calculated values [78]:

$$
f_{\mathrm{SRF}(\lambda / 4)} \approx \frac{\left(\frac{300}{4 l_{c}(1+0.225 d / l)}\right)^{0.8}}{\left(d^{2} / 73 p\right)^{0.2}}
$$




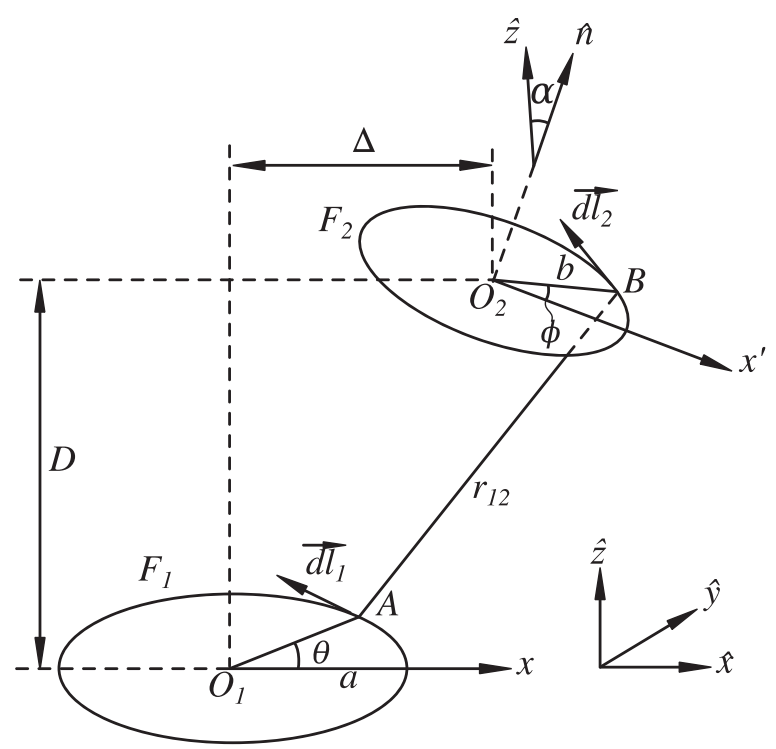

Fig. 10. General case of two coupled circular filaments $F_{1}$ and $F_{2}$, with coaxial, lateral, and angular displacement. Remastered from [80].

Equation (16) gives the quarter wave resonant frequency in MHz. It is important to note that (16) breaks down for coils with very few turns, since the approximation depends strongly on the pitch $p$ and the ratio $d / l$. Equation (16) assumes that $\epsilon \approx \epsilon_{0}$. By determining $f_{\mathrm{SRF}}$, it is possible to infer $C_{P}$ using the standard resonance approximation equation [79]:

$$
C_{P}=\left(\frac{1}{2 \pi f_{\mathrm{SRF}} \sqrt{L}}\right)
$$

Generally, the quarter-wave SRF of (16) can be considered to give the upper limit frequency for the usefulness of the lumped model. As a rule of thumb, $C_{P}$ can be completely neglected if the SRF is much greater $(10 \times$ or so) than the operating frequency.

\section{B. Link Modelling}

With each coil modelled in accordance with Fig. 6, the link itself must be modelled. This includes determining the mutual inductance of the link, as well as the gain, PTE, and PDL.

1) Mutual Inductance: A practical treatment of mutual inductance calculation is given by Soma and Galbraith, where each coil is considered as a series of circular filaments, each being coupled to all other filaments, dependent on their physical arrangement [80]. Fig. 10 shows the general case of two filaments $F_{1}$ and $F_{2}$ with radii $a$ and $b$, arranged with coaxial, lateral, and angular displacements, $D, \Delta$, and $\alpha$ respectively.

It is important to note that these mutual inductance calculations assume that each coil turn can be approximated as a thin filament, i.e. $r_{0} \ll r$. In the case of extremely compact coils [27], [81], the equations will progressively lose accuracy as $r_{0}$ approaches $r$.
The mutual inductance between these two filaments for purely coaxial displacement $(\Delta, \alpha=0, D \neq 0)$ is [80]:

$$
M_{\text {coax }}=\mu_{0} \sqrt{a b} G(\aleph),
$$

where

$$
\begin{aligned}
G(\aleph) & =\left(\frac{2}{\aleph}-\aleph\right) K(\aleph)-\frac{2}{\aleph} E(\aleph) \\
\aleph & =\sqrt{\frac{4 a b}{(a+b)^{2}+D^{2}}},
\end{aligned}
$$

where $K$ and $E$ are complete elliptic integrals of the first and second kind respectively. While the purely coaxial case is simple, it is more complex to describe the general case that includes lateral and angular displacements. Fortunately it is possible to separate the effects of lateral and angular misalignments, and consider them as essentially orthogonal [34], [80]. Soma presents expressions using numerical integration to calculate $M$ for these cases, based on [82]. For the lateral misalignment case $(\alpha=0, D, \Delta \neq 0)$ :

$$
M_{\text {lat }}=\frac{\mu_{0} a b}{2 \pi} \oint \frac{\cos \beta}{\sqrt{a b_{\text {lat }}}} G\left(\aleph_{\text {lat }}\right) d \phi,
$$

where

$$
\begin{aligned}
\aleph_{\text {lat }} & =\sqrt{\frac{4 a b_{\text {lat }}}{\left(a+b_{\text {lat }}\right)^{2}+D^{2}}}, \\
b_{\text {lat }} & =\sqrt{b^{2}+\Delta^{2}+2 \Delta b \cos \phi}, \\
\beta & =\tan ^{-1}\left(\frac{\Delta \sin \phi}{b+\Delta \cos \phi}\right) .
\end{aligned}
$$

For the angular misalignment case $(\Delta=0, D, \alpha \neq 0)$ :

$$
M_{\mathrm{ang}}=\frac{\mu_{0} \sqrt{a b}}{\pi \sqrt{\cos \alpha}} \int_{0}^{\pi}\left(\frac{\cos \lambda}{\cos \phi}\right)^{\frac{3}{2}} G\left(\aleph_{\mathrm{ang}}\right) d \phi,
$$

where

$$
\begin{aligned}
\aleph_{\text {ang }} & =\sqrt{\frac{\frac{4 a b \cos \phi}{\cos \lambda}}{a^{2}+b^{2}+D^{2}-2 b D \cos \phi \sin \alpha+\frac{2 a b \cos \phi \cos \alpha}{\cos \lambda}}}, \\
\lambda & =\tan ^{-1}\left(\frac{\sin \phi}{\cos \phi \cos \alpha}\right) .
\end{aligned}
$$

Equations (18), (21), and (25) can be used to determine the mutual inductance between individual filaments; the total mutual inductance can be determined through summation across all the turns of each coil [83]:

$$
M=\sum_{i=1}^{n_{1}} \sum_{i=1}^{n_{1}} M_{i j}(a, b, D, \Delta, \alpha) .
$$

This mutual inductance can also be considered as a unitless coupling coefficient $k$ :

$$
k=M / \sqrt{L_{1} L_{2}} .
$$




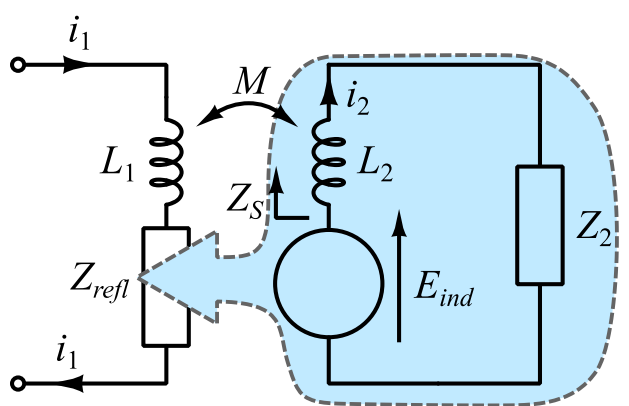

Fig. 11. Simplified coupled circuit reflects an impedance in series with the primary.

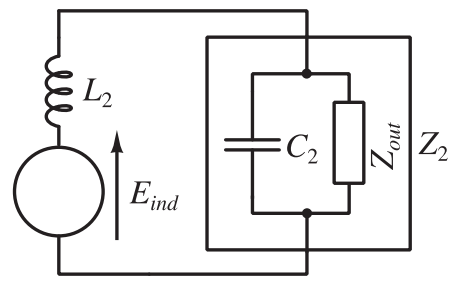

Fig. 12. Example contents of $Z_{2}$ (see Fig. 11) for a parallel resonant secondary coil.

Since $k$ is normalized to the mean inductance of the two link coils, $k$ values can be freely compared between links of arbitrary geometry.

2) Link Impedance: The previous sections have focused on converting geometric parameters to electrical parameters, aiming to determine all the parameters in Table II. These electrical parameters can then be used to model the link in accordance with Fig. 2. The element to consider first is the coupling of the secondary circuit into the primary, in order to determine the impedance presented by the link to the driver. The principle is described in detail by Terman as follows [29].

The current flowing in the primary coil will induce a voltage in series with the secondary coil, which is experienced as a 'reflected load' at the primary side. The load is defined as

$$
Z_{\text {refl }}=\frac{(\omega M)^{2}}{Z_{S}},
$$

where $Z_{S}$ is the impedance seen by the induced voltage $E_{\text {ind }}$, which is defined as

$$
E_{\text {ind }}=-j \omega M i_{1}=i_{2} Z_{S} .
$$

The principle is illustrated in Fig. 11; the secondary side of the link can be transformed into the reflected impedance $Z_{\text {refl }}$ in series with the primary coil. Using this process, the link impedance, gain, and efficiency can be readily calculated. For instance, the link impedance in the simplified case (as in Fig. 11) is given by:

$$
Z_{\text {link (simple })}=j \omega L_{1}+\frac{(\omega M)^{2}}{j \omega L_{2}+Z_{2}},
$$

TABLE III

LINK IMPEDANCES IN ACCORDANCE WITH FIGS. 2 \& 3

\begin{tabular}{cc}
\hline Topology & Link Impedance $Z_{\text {link }}$ \\
\hline $\mathrm{SS}$ & $Z_{L 1}+\frac{1}{j \omega C_{1}}+\frac{(\omega M)^{2}}{Z_{L 2}+\frac{1}{j \omega C_{2}}+Z_{\text {out }}}$ \\
\hline $\mathrm{SP}$ & $Z_{L 1}+\frac{1}{j \omega C_{1}}+\frac{(\omega M)^{2}}{Z_{L 2}+\frac{1}{j \omega C_{2}+1 / Z_{\text {out }}}}$ \\
\hline $\mathrm{PS}$ & $\left(j \omega C_{1}+\frac{1}{Z_{L 1}+\frac{1}{Z_{L 2}+\frac{1}{j \omega C_{2}}+Z_{\text {out }}}}\right)^{-1}$ \\
\hline $\mathrm{PP}$ & $\left(j \omega C_{1}+\frac{1}{Z_{L 1}+\frac{1}{Z_{L 2}+\frac{1}{j \omega C_{2}+1 / Z_{\text {out }}}}}\right)^{-1}$
\end{tabular}

where $Z_{2}$ is the impedance seen by the coil $L_{2}$, usually formed of the resonating capacitor and output load $Z_{\text {out }}$ (see Fig. 12). For the four NRIC configurations as in Fig. 2, expressions for $Z_{\text {link }}$ are given in Table III, where $Z_{L 1}$ and $Z_{L 2}$ are the impedances of the coils including their parasitics, in accordance with Fig. 6:

$$
Z_{L 1, L 2}=\left(\frac{1}{j \omega L_{1,2}+R_{1,2}}+j \omega C_{P 1, P 2}\right)^{-1} .
$$

If the operating frequency is well below the SRF of both coils, (33) can be simplified to:

$$
Z_{L 1, L 2}=j \omega L_{1,2}+R_{1,2} .
$$

By substituting coil impedances from (33) or (34) into the expressions in Table III, $Z_{\text {link }}$ can be calculated. With $Z_{\text {link }}$ in place, gain, power, and efficiency figures can be determined.

3) Gain: As discussed in Section III-A, the link gain will either be a voltage gain or a transimpedance, depending on the primary tank configuration and primary driver. It is assumed that series-resonant primaries are driven by voltage sources and parallel-resonant primaries are driven by current sources. In both cases, the voltage output $v_{\text {out }}$ (see Fig. 3) can be defined in terms of the induced voltage $E_{\text {ind }}$, for SS and PS links:

$$
v_{\text {out }}=\frac{E_{\text {ind }} Z_{\text {out }}}{Z_{L 2}+\frac{1}{j \omega C_{2}}+Z_{\text {out }}},
$$

and for SP and PP links:

$$
v_{\text {out }}=\frac{E_{\text {ind }}}{Z_{L 2}\left(j \omega C_{2}+1 / Z_{\text {out }}\right)+1} .
$$

Since $E_{\text {ind }}$ can be determined in terms of the current $i_{1}$ flowing into $Z_{\text {reff }}$, see (31), gains can be defined by expressing $i_{1}$ in terms of the input voltage or current. For SS and SP links:

$$
i_{1}=\frac{v_{\text {in }}}{Z_{\text {link }}},
$$

and for PS and PP links:

$$
i_{1}=\frac{i_{\text {in }} Z_{\text {link }}}{Z_{L 1}+Z_{\text {refl }}} .
$$


Therefore, the link gains for all four configurations can be defined:

$$
\begin{aligned}
& A_{S S}=\frac{v_{\text {out }}}{v_{\text {in }}}=\frac{-j \omega M Z_{\text {out }}}{Z_{\text {link }}\left(Z_{L 2}+\frac{1}{j \omega C_{2}}+Z_{\text {out }}\right)}, \\
& A_{S P}=\frac{v_{\text {out }}}{v_{\text {in }}}=\frac{-j \omega M}{Z_{\text {link }}\left(Z_{L 2}\left(j \omega C_{2}+\frac{1}{Z_{\text {out }}}\right)+1\right)}, \\
& A_{P S}=\frac{v_{\text {out }}}{i_{\text {in }}}=\frac{-j \omega M Z_{\text {out }} Z_{\text {link }}}{\left(Z_{L 1}+Z_{\text {refl }}\right)\left(Z_{L 2}+\frac{1}{j \omega C_{2}}+Z_{\mathrm{out}}\right)}, \\
& A_{P P}=\frac{-j \omega M Z_{\text {link }}}{\left(Z_{L 1}+Z_{\text {refl }}\right)\left(Z_{L 2}\left(j \omega C_{2}+\frac{1}{Z_{\text {out }}}\right)+1\right)} .
\end{aligned}
$$

4) PDL and PTE: Since the output voltage $v_{\text {out }}$ is defined in (35) and (36) as being across $Z_{\text {out }}$, the true power delivered to the load can be written simply as

$$
P_{\text {out }}=\frac{\left|v_{\text {out }}\right|^{2} \mathfrak{R}\left\{Z_{\text {out }}\right\}}{\left|Z_{\text {out }}\right|^{2}} .
$$

$P_{\text {out }}$ will be maximized in the case that both $v_{\text {out }}$ and $Z_{\text {out }}$ are purely real, i.e. the power factor is unity. If, for instance, the drive frequency differs from the resonant frequency of the receiving tank, $v_{\text {out }}$ will have a phase lag/lead, which must be compensated for by adding a matching reactance to $Z_{\text {out }}$. Of course, the load matching theorem also applies; for SS and PS links where the receiving tank is resonating, $Z_{\text {out }}$ should equal $R_{2}$, and for SP and PP links where the receiving tank is resonating $Z_{\text {out }}$ should equal the parallel resistance of the receiving tank $\left(R_{P 2}\right)$. The parallel resistance of a coil can also be defined in terms of its series resistance and Q-factor (see (15) and Fig. 6):

$$
R_{P}=R\left(Q^{2}+1\right) .
$$

Calculating PTE is considered in detail in [42], where the link efficiency is considered in two parts: the efficiency from the transmitter to $Z_{\text {refl }}, \eta_{1}$, and the efficiency from the receiver to $Z_{\text {out }}, \eta_{2}$. Van Schuylenbergh assumes that both tanks are tuned to the operating frequency for the purpose of simplification [42], this is not assumed here for completeness. These efficiencies are defined as follows:

$$
\begin{aligned}
& \eta_{1}=\frac{P_{Z_{\text {refl }}}}{P_{\text {in }}}=\frac{\left|i_{1}\right|^{2} \Re\left\{Z_{\text {refl }}\right\}}{\left|i_{\text {in }}\right|^{2} \Re\left\{Z_{\text {link }}\right\}}=\frac{\left|i_{1}\right|^{2} \mathfrak{R}\left\{Z_{\text {refl }}\right\}\left|Z_{\text {link }}\right|^{2}}{\left|v_{\text {in }}\right|^{2} \Re\left\{Z_{\text {link }}\right\}}, \\
& \eta_{2}=\frac{P_{\text {out }}}{P_{Z_{\text {refl }}}}=\frac{\left|v_{\text {out }}\right|^{2} \Re\left\{Z_{\text {out }}\right\}}{\left|i_{1}\right|^{2} \Re\left\{Z_{\text {refl }}\right\}\left|Z_{\text {out }}\right|^{2}} .
\end{aligned}
$$

Equations (45) and (46) can be combined to determine the total link efficiency:

$$
\eta_{1} \eta_{2}=\frac{P_{Z_{\text {refl }} P_{\text {out }}}}{P_{\text {in }} P_{Z_{\text {refl }}}}=\frac{P_{\text {out }}}{P_{\text {in }}}=\eta_{\text {link }} .
$$

Considering the behavior of the link intuitively, if the coupling and load are such that $Z_{\text {refl }}$ appears real, and both coils have high Q-factors $\left(R_{1,2} \rightarrow 0\right)$, then $\eta_{\text {link }}$ will approach unity, assuming the power source is also impedance matched to $Z_{\text {link }}$. This behavior can be formalized in terms of the kQ product [84][86]:

$$
k \sqrt{Q_{1} Q_{2}} \text {. }
$$

The $Q$ part of the product is the geometric mean of the Qfactors of the two coils. The maximum theoretical efficiency can be shown to be directly proportional to (48) [85]:

$$
\eta_{\operatorname{link}_{(\max )}}=\frac{k^{2} Q_{1} Q_{2}}{\left(1+\sqrt{1+k^{2} Q_{1} Q_{2}}\right)^{2}} .
$$

\section{Limitations of Analytic Modelling}

It is important to note that the analytic modelling techniques discussed in the previous subsections rely on the assumption that $\mu=\mu_{0}$ and $\epsilon=\epsilon_{0}$ everywhere in the system. As a result, they can be insufficient for modelling inductive links in complex environments containing combinations of tissue, electronics, epoxies, and other materials, all with different $\mu$ and $\epsilon$ values. Analytic methods can still be used to model simple muscular environments with good results however. For example, Jow et al. demonstrate errors of only a few percent between calculation and simulation for free space and muscle environments [31]. For these kinds of calculation, it is useful to note that, at lower frequencies, biological tissue can be considered to have $\mu=\mu_{0}$; $\epsilon$ however is more variable, and particularly dependent on tissue type [87], [88]. For environments containing layers of materials with many different permittivities, analytic calculations become more difficult to perform; as a result, therefore FEM simulations or physical measurements may be necessary in later design iterations if precise results are required.

\section{CONSIDERATIONS AND TRADEOFFS}

When undertaking a link design, constraints and targets must be considered. Common constraints include the geometry, materials, and operating frequency; common targets include PTE, PDL, and misalignment tolerance. Considering the flow diagram in Fig. 4, the main iteration is in coil geometry, as the geometry determines the electrical parameters, and thus the link performance. It is therefore crucial for the designer to understand how to adjust geometric parameters in order to obtain the desired change in electrical parameters. This section reviews a number of factors that should be considered during this process, describes relations between geometric and electrical parameters, and discusses how to determine whether design targets are feasible or not.

\section{A. Frequency Splitting}

The previous analysis in Section III has implied that, in an NRIC link, both tanks should be tuned to the same frequency. This intuitively appears to be the best approach to maximize PDL and PTE, because if both tanks are driven at their resonant frequencies, they will appear to be real. The problem with this assumption is that it does not account for the fact that $Z_{\text {refl }}$ 


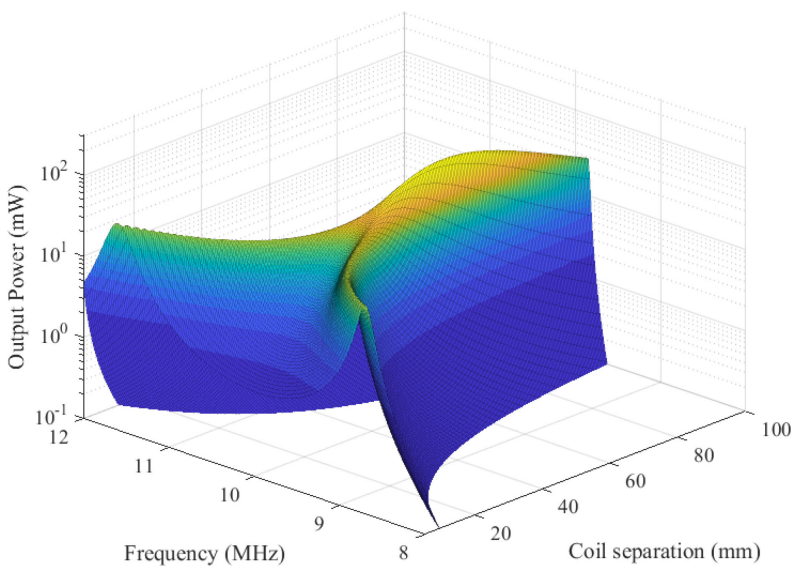

Fig. 13. Frequency splitting in power output from an example SS link driven by a $1 \mathrm{~V}$ source.

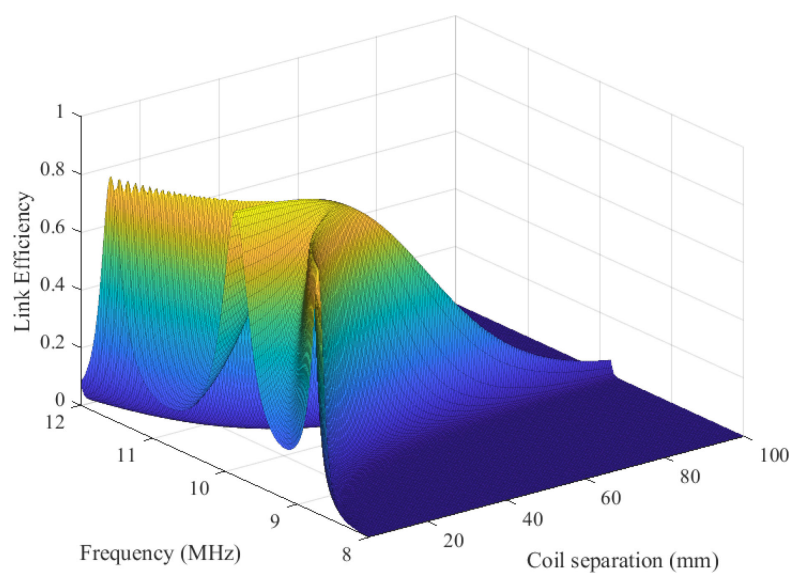

Fig. 14. Frequency splitting in efficiency output for an example SS link driven by a $1 \mathrm{~V}$ source.

appears as a complex impedance in series with the primary coil, whose value is dependent on the coupling. This manifests in the form of frequency-splitting, also known as pole-splitting, where the optimum drive frequency of the link varies from the tuned frequency as the coils are brought closer together [45], [89]-[95]. The effect can be observed directly by plotting parameters such as the link gain, impedance, and efficiency against changes in drive frequency and coupling. Figs. 13 and 14 show the link efficiency and power output for an example link in SS configuration driven by an ideal $1 \mathrm{~V}$ source, in accordance with (43) and (47). The link specifications that are independent of frequency and coupling are given in Table IV; both coils are identical wire solenoids, arranged in coaxial alignment $(\Delta, \alpha=$ $0)$. Figs. 13 and 14 clearly show that, despite both sides of the link being tuned to resonate at $10 \mathrm{MHz}$, reducing the separation distance to below $40 \mathrm{~mm}$ induces splitting in both the PDL and PTE responses. While it is possible to maintain a high PTE with a fixed frequency of $10 \mathrm{MHz}$ up to very short spacing distances (see Fig. 14), the accompanying PDL drops significantly (see Fig. 13). It should be noted that the efficiency plot in Fig. 14
TABLE IV

EXAMPLE LINK SPECIFICATIONS

\begin{tabular}{cc}
\hline Parameter & Value \\
\hline Coil diameter $\left(d_{1,2}\right)$ & $40 \mathrm{~mm}$ \\
\hline Wire diameter $\left(d_{0(1,2)}\right)$ & $0.4 \mathrm{~mm}$ \\
\hline Winding pitch $\left(p_{1,2}\right)$ & $0.6 \mathrm{~mm}$ \\
\hline Number of turns $\left(n_{1,2}\right)$ & 10 \\
\hline Inductance $\left(L_{1,2}\right)$ & $7.1 \mu \mathrm{H}$ \\
\hline Capacitance $\left(C_{1,2}\right)$ & $35.7 \mathrm{pF}$ \\
\hline Tuned frequency $\left(f_{0(1,2)}\right)$ & $10 \mathrm{MHz}$ \\
\hline Output load $\left(Z_{\text {out }}\right)$ & $20 \Omega$
\end{tabular}

assumes the link is being driven by an ideal source; for a real source, the efficiency at the center frequency will drop due to the change in $Z_{\text {link }}$. For links with variable separation, frequency splitting must be considered if both high PTE and PDL are to be maintained. Splitting is generally tackled by implementing an adjustable frequency drive to track the optimum frequency [37], [94], [95], or adjustable link components to force the optimum frequency back to the drive frequency [12], [89], [93].

The exact optimum frequencies can be determined by partial differentiation. For instance, solving

$$
\left.\frac{\partial P_{\text {out }}}{\partial \omega}\right|_{M_{\min }<M<M_{\max }}=0,
$$

(where $M_{\min }<M<M_{\max }$ describes the desired range of mutual inductances) will produce a series of positive real root frequencies; odd roots correspond to peaks and even roots correspond to troughs. Equation (50) can be most easily solved through symbolic mathematical software in order to find the root paths. An alternative approach is given by Niu et al. [37], where direct expressions are given for root paths for an 'SS' link. By controlling a variable frequency drive in accordance with odd roots from (50), the power output can be maximized regardless of coil separation.

\section{B. Maximizing the $k Q$ Product}

Considering the $k Q$ product from (48) as a figure of merit, an ideal link can be considered as one with a $k Q$ product that tends to infinity. Adjusting geometric parameters with the aim of maximizing $k Q$ however is largely an exercise in tradeoffs. For instance, Fig. 15 shows the effect of adjusting the winding pitch of a solenoid (with otherwise the same geometry as in Table IV) on its Q-factor and length. By packing the windings as close as possible, the Q-factor will be suppressed by proximity effect losses, but if the pitch is too large, the Q-factor drops due to reduced inductance. In this example, to maximize $Q$ by increasing the pitch requires an increase in the coil length of $2.5 \times$, which will have a detrimental effect on the coupling $k$, assuming the edge to edge separation D remains the same. This is illustrated in Fig. 16; increasing the pitch effectively increases the mean distance between turns, reducing the coupling coefficient. Maintaining a winding pitch that is greater than the minimum can also pose a practical problem; the coil must be constructed 


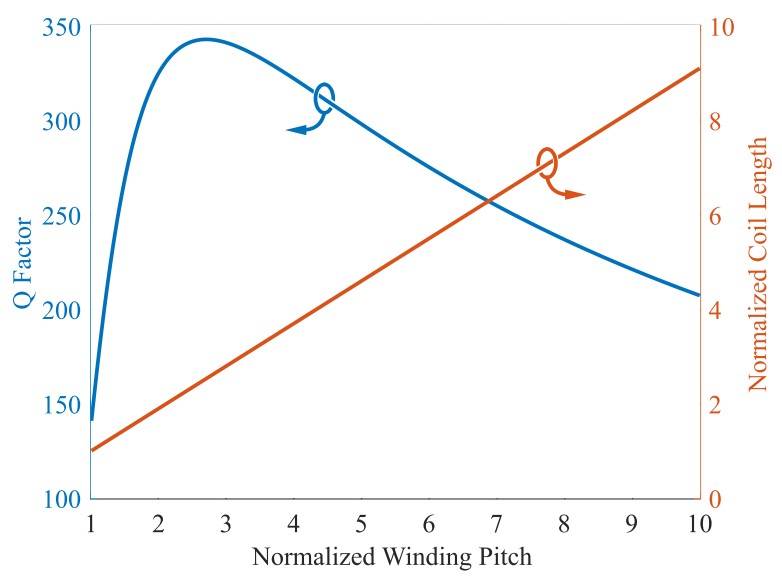

Fig. 15. Optimizing winding pitch to maximize $Q$ comes at the cost of increased coil length. Pitch is normalized to the minimum pitch $p=d_{0}$.

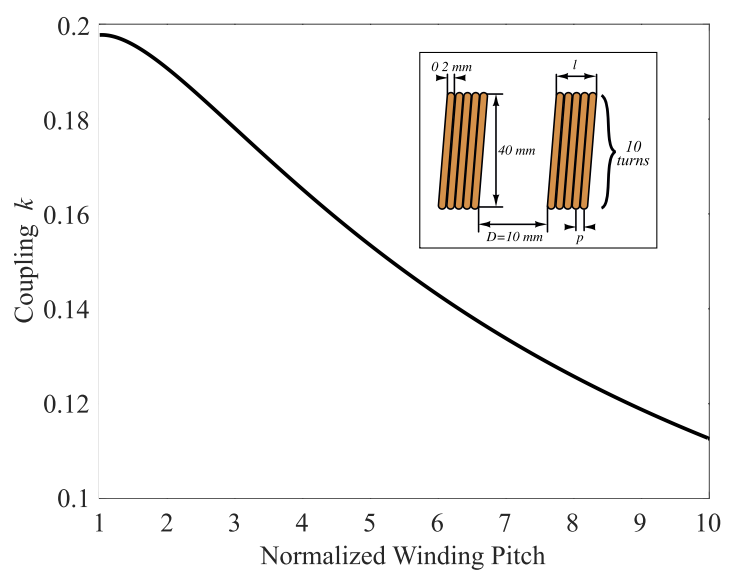

Fig. 16. Decrease in coupling coefficient between two identical coils as winding pitch is modified.

with a former or glue such that the desired pitch is maintained. This is not a problem for PCB based coils; the tracks are fixed in place on the substrate, guaranteeing the desired pitch.

It is important to note also that Q-factor optimization by pitch adjustment is affected by operating frequency. This is particularly important to consider in variable frequency drive systems, as it will dictate the optimum splitting root path to follow. This can be seen in Figs. 13 and 14; the lower frequency splitting path gives slightly higher power output and efficiency figures than the higher frequency path, due to the dependence of Q-factor on frequency. The effect of frequency on Q-factor is further highlighted in Fig. 17; as the operating frequency increases, the optimum winding pitch also increases.

Similar to varying the winding pitch, varying the number of turns in each coil also reveals tradeoffs. Considered qualitatively, increasing the number of turns has the effect of increasing the length, inductance, and Q-factor up to a limit. It also effects the SRF. Fig. 18 shows how increasing the number of turns increases the Q-factor, up until the point at which the increased losses outweigh the increased inductance. Similar to the case of winding pitch, the increase in length also causes a decrease in

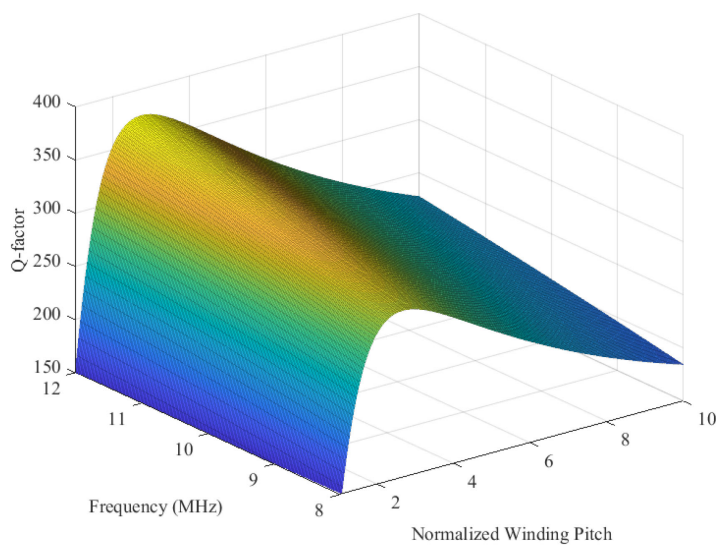

Fig. 17. Decrease in coupling coefficient between two identical coils as winding pitch is modified, with respect to frequency.

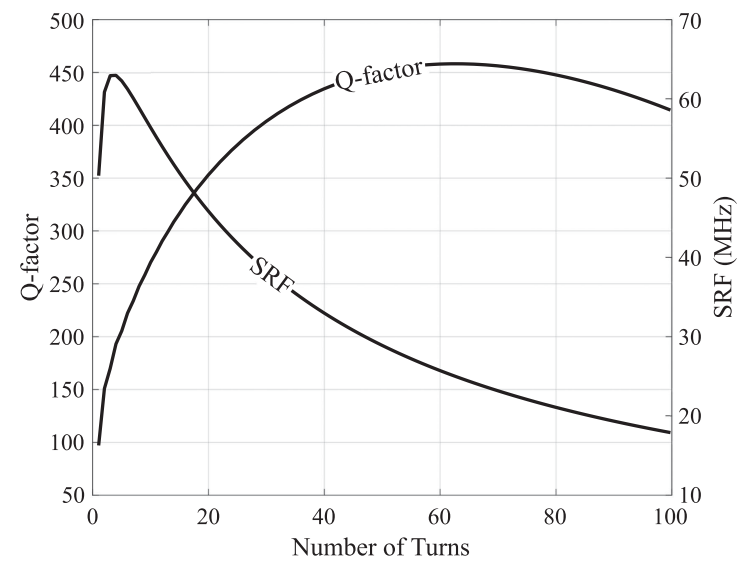

Fig. 18. Effect of increasing the number of turns on Q-factor and SRF. Parameters otherwise the same as in Table IV.

$k$, so it is better to adjust turns aiming to maximize $k Q$, rather than just the Q-factor. Since increasing the number of turns also reduces the SRF, the point at which the SRF approaches the operating frequency can be considered a hard limit on the allowed number of turns in either coil.

\section{Displacement Resilience}

Resilience to displacement, particularly in the lateral and angular sense, is a target that depends strongly on application. In cochlear implants for instance, link coaxial alignment can be assumed, as the coils are magnetically aligned [96]. In the case of spectacle-mounted retinal stimulator links however, this alignment cannot be guaranteed [97]. In such cases, it is more desirable to ensure that an inductive link is resilient to displacement variations than solely focusing on maximizing PTE and PDL. A simple method of improving displacement tolerance is described by Donaldson and Perkins [33], based on the work of Ko et al. [32], where it is suggested that by determining a ratio of coil diameters that maximizes $k$ for a given $D$, resilience to displacement in general will be maximized. This conclusion can be explained by the fact that any displacements, axial, lateral, 
or angular, will reduce $k$, and so by maximizing $k$ for a given arrangement, this reduction can be mitigated. Ko gives the ideal transmitter coil diameter $d_{1}$ for maximum resilience in terms of a given receiver coil diameter $d_{2}$ and coaxial distance $D$ [32]:

$$
d_{1}=\sqrt{d_{2}^{2}+4 D^{2}}
$$

In addition to resilience considerations, since $k$ is maximized by selecting diameters in accordance with (51) the $k Q$ product is increased, boosting PTE and PDL.

By increasing the ratio $d_{1} / d_{2}$ even further, $k$ can be kept very stable against displacements, at the cost of the absolute value of $k$. This strategy is employed in wireless powering of endoscopic capsules, where the receiver is subject to significant movement, and PTE and PDL are not inherently critical [98]-[100].

Adaptive systems are also popular for improving displacement tolerance. Figs. 13 and 14 show how coupling changes due to displacement can result in a change in optimum frequency; by adapting the operating frequency or resonant capacitance, such systems are more resilient to these changes in displacement [12], [15], [39], [40], [101], [102].

\section{Selecting a Resonant Topology}

In addition to optimizing the coil geometry for a given application, a resonant topology that is appropriate to the application must also be selected. Each of the four resonant topologies as shown in Fig. 2 suits a different application, since each topology presents a different impedance. The problem of topology selection has been considered in the literature, with differing conclusions about when to use series- and parallel-resonant topologies [36], [103], [104].

Considering the link as two coupled resonant tanks, the appropriate topology can be determined by observing the impedance of each tank relative to the impedance of the power amplifier and the receiver, and matching them. In Fig. 2, series-resonant primaries are driven by voltage sources, and parallel-resonant primaries by current sources for this reason. The principle holds when considering the secondary side of the link; for high output impedances, a parallel-resonant secondary will improve PTE and PDL, and vice-versa. This difference is demonstrated in Fig. 19; an otherwise identical link will have very different optimum load conditions depending on its resonant topology. Here the SP link works best for a $Z_{\text {out }}$ that is 3 orders of magnitude greater than the optimum $Z_{\text {out }}$ for the SS configuration.

It is for this reason that the SP link configuration is common for biomedical inductive links [4], [8], [33], [105], [106]. The receiver will commonly have a relatively high input impedance, and the driver will often be a Class-D or -E amplifier, suited to driving low impedances [23], [97], [98], [107]. If maximizing PTE and PDL are crucial, it is possible to determine the optimum load impedance for a given topology analytically [108]. This analysis has been adopted for the design of adaptive rectification circuits, that aim to present an optimum impedance to the link at all times by varying the rectification parameters (duty cycle, frequency etc.) [109]-[111].

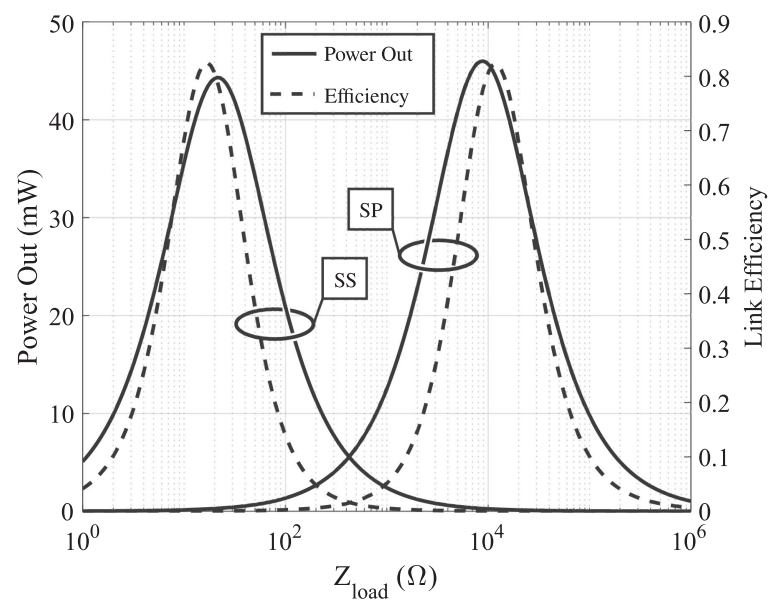

Fig. 19. Curves of PDL and PTE for SS and SP configurations as $Z_{\text {out }}$ is varied. Parameters are otherwise the same as in Table IV. $D=40 \mathrm{~mm}$.
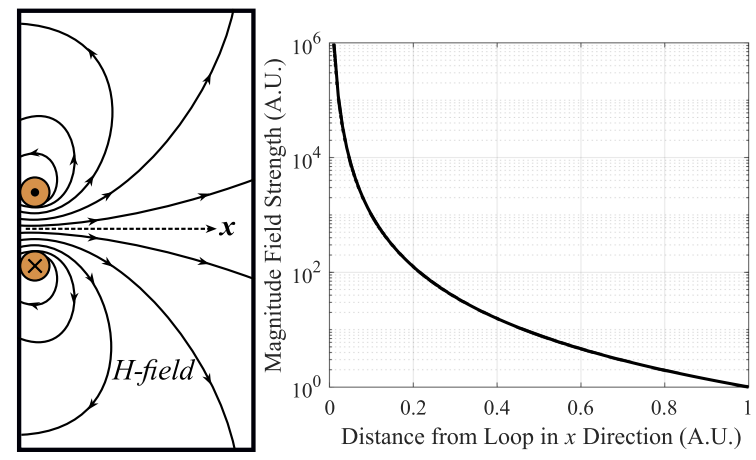

Fig. 20. Left: magnetic flux lines from an energized loop of wire, right: field strength for increasing distance from the loop in coaxial alignment.

\section{E. Refining Targets}

During the design process, it may become apparent that the design targets are impossible to achieve given the initial design constraints. For example, if both coils are limited to a diameter of $10 \mathrm{~mm}$, and the target axial distance is $50 \mathrm{~mm}$, it will be impossible to achieve a high PDL and PTE. The triad inset in Fig. 4 qualitatively shows three desirable design features that must be traded off against each other. At most, two out of three of the features can be achieved; sacrificing one feature will often improve the other two. This triad should be considered when determining an initial link specification, in order to ensure that the desired targets are reasonable given the constraints. When trying to determine if a target coil separation distance is reasonable, a useful rule to remember is the fact that the magnetic field strength from an energized coil decays proportional to the inverse cube of the coaxial distance from its center. This is illustrated in Fig. 20; the current- carrying loop is essentially a magnetic dipole, and shows the characteristic divergence in its flux lines as distance from the loop increases. The number of flux lines captured by the receiving coil will determine the coupling coefficient $k$ between the two coils. Since NRIC doesn't require extremely high coupling for high PTE and PDL, the coils don't 


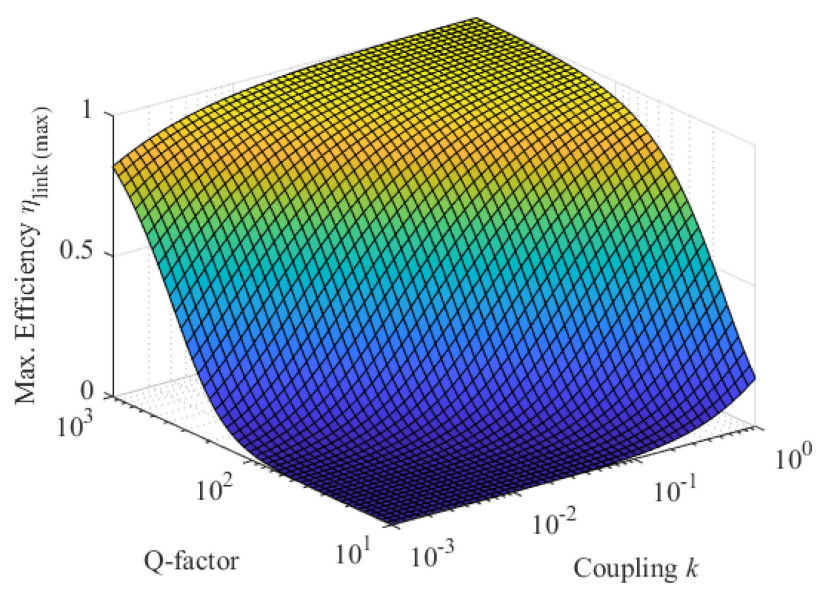

Fig. 21. Maximum theoretical link efficiency (according to (49)), as the coupling coefficient and average (geometric) Q-factor are varied.

have to be extremely close to each other, as in non-resonant coupling.

The exact minimum value of $k$ to maintain a given target value of PTE therefore depends more on the average $Q$ of the two coils. Fig. 21 shows how the maximum theoretical PTE can remain high even for very low coupling coefficients, providing the average Q-factor is high. The maximum obtainable Q-factor will depend on the available materials, operating frequency, dimensions etc, as discussed in the previous sections.

This principle also applies when considering coil size and displacement tolerance. Smaller coils will generally have a lower $Q$ and worse overall displacement tolerance; the smaller coil size makes the loop appear less like a dipole.

\section{Software AND Design EXAMPLE}

To automate some of the processes in Fig. 4, the Coupled Coil Configurator (CuCCo) software has been developed for MATLAB [43]; an early version of the software is currently available for download, is in active development at the time of writing, and is licensed under the GNU GPLv3 [112]. Feature requests and contributions are encouraged. The aim of $\mathrm{CuCCo}$ is to provide functions that perform geometric to electrical parameter conversion, and link performance prediction purely through analytical calculation. The motivation behind creating $\mathrm{CuCCo}$ was to provide a single tool that fills the gap between spice-like circuit simulation and FEM solver software. Instead of having to simulate coil geometries in FEM and convert them into electrical parameters for circuit simulation and link characterization, $\mathrm{CuCCo}$ aims to provide both functions with less overhead, by employing an analytic approach. The methods in this paper are mostly included in $\mathrm{CuCCo}$, to obviate the need for the reader to re-implement them themselves. The plots throughout this paper have all been generated using calculated results from $\mathrm{CuCC}$. The following section is a design example, to demonstrate some of the capabilities of the software, and to show how
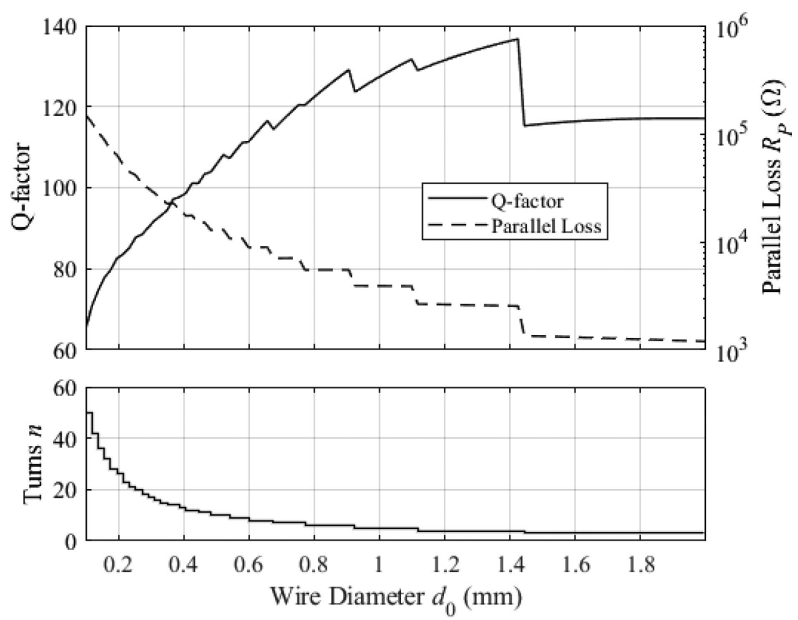

Fig. 22. Finding optimum Q-factor as wire diameter is varied, following diameter and length constraints.

to employ the principles in Sections III and IV in an example application.

\section{A. Design Example: Cochlear Implant}

1) Constraints and Calculations: Typical cochlear implants require around $40 \mathrm{~mW}$ of power at the receiver, to be delivered through the skull by small coils aligned with a permanent magnet [96]. For this example, both coils should have diameters no larger than $20 \mathrm{~mm}$, and should be within $4-6 \mathrm{~mm}$ in length. Typical temporal skull thicknesses range from 6-12 mm [113]; the link should therefore function for coaxial $D$ values in this range. Finally, PTE is a high priority for this example; higher PTE translates to a longer battery life, which is beneficial for the patient. For simplicity, a fixed-frequency non-adaptive driver running in the ISM band at $6.78 \mathrm{MHz}$ is assumed. The receiving coil should feed a rectifier and produce at least 13 $\mathrm{V}$ (dc), to provide $12 \mathrm{~V}$ after regulation, for the stimulator circuit.

To begin, the requirements of the receiver should be considered. If the implant requires $13 \mathrm{~V}$, and will draw a maximum of $40 \mathrm{~mW}$, this can be translated to a load:

$$
R_{i m p l}=\frac{V^{2}}{P}=\frac{13^{2}}{40 \times 10^{-3}}=4225 \Omega .
$$

Equation (52) gives the load presented by the implant after the rectifier. A simple method of including the effect of the rectifier is given by Donaldson and Perkins [33], where the equivalent load seen by the link can be considered in terms of $R_{i m p l}$ :

$$
R_{L}=\frac{R_{i m p l}}{2} \approx 2113 \Omega
$$

Given this high value of $R_{L}$, a parallel resonant secondary is most appropriate for impedance matching (see Fig. 19). The next step is to determine the optimum geometry given the constraints. Fig. 22 shows the Q-factor, parallel loss resistance, 


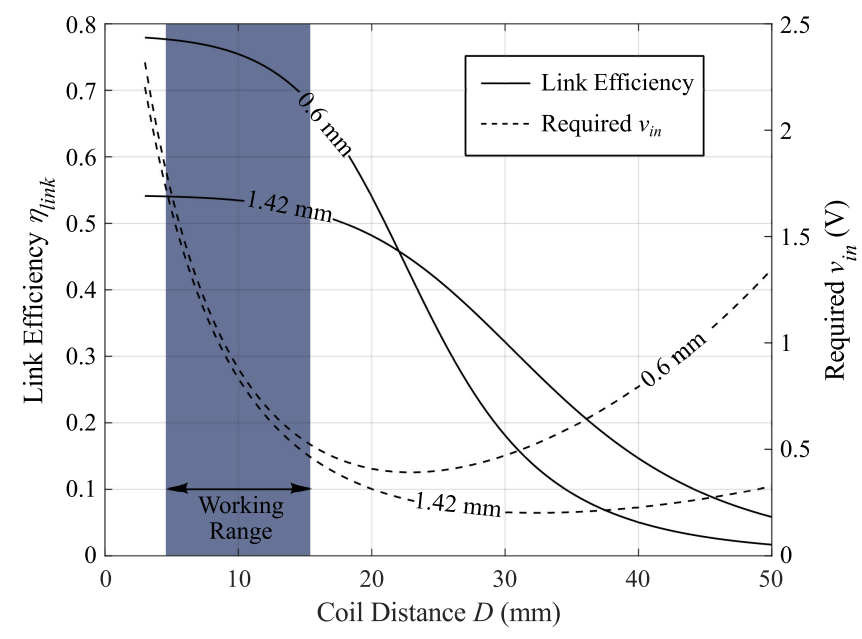

Fig. 23. Curves to show link efficiency and the required input voltage to achieve $40 \mathrm{~mW}$ output from an SP configured link, coil geometries in accordance with the text and Fig. 22, wire diameters noted on the curves.

and number of turns against changes in wire diameter for a single-layer solenoid, with minimum pitch, and its length limited to the $5 \mathrm{~mm}$ constraint. The number of turns is maximized to fill this length constraint. It should be noted that the coil series loss is assumed to have $50 \mathrm{~m} \Omega$ added in series, to include losses from connecting PCB traces, solder joints etc. This small additional loss causes the drop in Q- factor as $d_{0}$ rises above $1.4 \mathrm{~mm}$ in Fig. 22. The parallel loss resistance $R_{P}$ can be computed from the series loss resistance $R_{S}$ and the quality factor $Q$ :

$$
R_{P} \approx Q^{2} R_{S}
$$

It follows from the maximum power transfer theorem that when $R_{L}=R_{P}$, the output power will be maximized. Fig. 22 shows that to maximize PDL the optimum wire diameter is $1.42 \mathrm{~mm}$; where the Q-factor is approximately 136 , the coil has 4 turns, and the parallel loss is approximately $2.5 \mathrm{k} \Omega$. While this receiver coil geometry maximizes PDL, the PTE will be limited to $\approx 50 \%$ since $R_{P} \approx R_{L}$. To increase the PTE therefore, the wire diameter should be reduced to a value that yields a higher parallel loss, without losing too much Q-factor. Reducing the wire diameter down to $0.6 \mathrm{~mm}$ for instance gives a $350 \%$ improvement in $R_{P}\left(R_{P}=8.85 \mathrm{k} \Omega\right)$, at the cost of a $23 \%$ drop in $\mathrm{Q}$-factor $(Q=111)$. Since the coils can be assumed to have good coaxial alignment, identical Tx and Rx coil geometries are suitable. Fig. 23 shows the link efficiency difference between the $0.6 \mathrm{~mm}$ and $1.42 \mathrm{~mm}$ wire diameter coils, when configured as an SP link, as well as the input voltage required to maintain $40 \mathrm{~mW}$ in the $2.1 \mathrm{k} \Omega$ load. Since the relevant distances for this example are $6-12 \mathrm{~mm}$, the $0.6 \mathrm{~mm}$ coils show much better performance, maintaining link efficiency from $73.6 \%$ to $77.3 \%$ over this range. By comparison, the $1.42 \mathrm{~mm}$ coils achieve a maximum efficiency of only $54 \%$ at $6 \mathrm{~mm}$ separation. In both cases, an input voltage of $0.5 \mathrm{~V}-2.0 \mathrm{~V}$ is required; this can be implemented as a fixed $2.0 \mathrm{~V}$ input voltage, which
TABLE V

COCHLEAR IMPLANT EXAMPLE LINK SPECIFICATIONS

\begin{tabular}{cc} 
Parameter & Value \\
\hline Coil diameter $\left(d_{1,2}\right)$ & $20 \mathrm{~mm}$ \\
\hline Wire diameter $\left(d_{0(1,2)}\right)$ & $0.5 \mathrm{~mm}$ \\
\hline Winding pitch $\left(p_{1,2}\right)$ & $0.6 \mathrm{~mm}$ \\
\hline Number of turns $\left(n_{1,2}\right)$ & 8 \\
\hline Inductance $\left(L_{1,2}\right)$ & $1.87 \mu \mathrm{H}$ \\
\hline Capacitance $\left(C_{1,2}\right)$ & $295 \mathrm{pF}$ \\
\hline Tuned frequency $\left(f_{0(1,2)}\right)$ & $6.78 \mathrm{MHz}$ \\
\hline Output load $\left(Z_{\text {out }}\right)$ & $2 \mathrm{k} \Omega$ \\
\hline
\end{tabular}

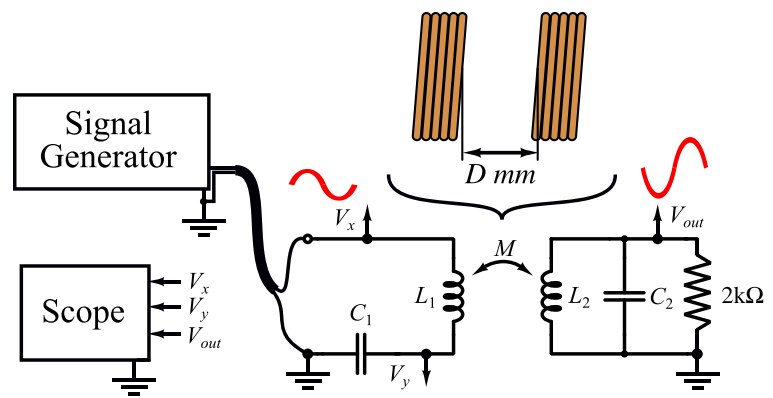

Fig. 24. Diagram of the test arrangement, measured voltages are annotated.

can then be regulated at the receiver side. Alternatively, if the Rx can communicate with the Tx, the Rx can command the Tx to increase, decrease, or maintain the input voltage to perform regulation.

2) Experimental Validation: The proposed design was manufactured and measured to validate the predictions in the previous section. The measured parameters of the manufactured coils are given in Table V. In this example, $0.5 \mathrm{~mm}$ diameter wire was used instead of $0.6 \mathrm{~mm}$, but the pitch was maintained at $0.6 \mathrm{~mm}$. The measured Q-factor of 127 shows a good correlation with the predicted $Q$ of 115 from Fig. 22. The link was arranged as shown in Fig. 24, with the coils in coaxial alignment, separated by $D \mathrm{~mm}$. The signal generator used was an Agilent 33250A, and the oscilloscope was an Agilent MSO6104A. $V_{\text {out }}$ was measured to maintain a constant voltage of $9.0 \mathrm{~V}$ (p-p), translating to $40 \mathrm{~mW}$ (p-p) at the output. All voltages in the testing process are sinusoid signals. By measuring the voltages $V_{x}$ and $V_{y}, i_{1}$, and $Z_{\text {refl }}$. Using these, $\eta_{1}$ can be determined.

$$
\begin{aligned}
i_{1} & =\frac{V_{y}}{Z_{C_{1}}}=\frac{V_{x}-V_{y}}{Z_{L_{1}}+Z_{\text {refl }}} \\
\therefore Z_{\text {refl }} & =\frac{V_{x}-V_{y}}{i_{1}}-Z_{L_{1}} .
\end{aligned}
$$

Since $Z_{\text {link }}=Z_{L_{1}}+Z_{C_{1}}+Z_{\text {reff }}$, the results from (55) and (56) can be applied to (45) to determine $\eta_{1}$. Since the link is being operated at the resonant frequency of the Rx (and the Tx), it is simpler to determine $\eta_{2}$ by using the measured $Q$ of the $\mathrm{Rx}$, 


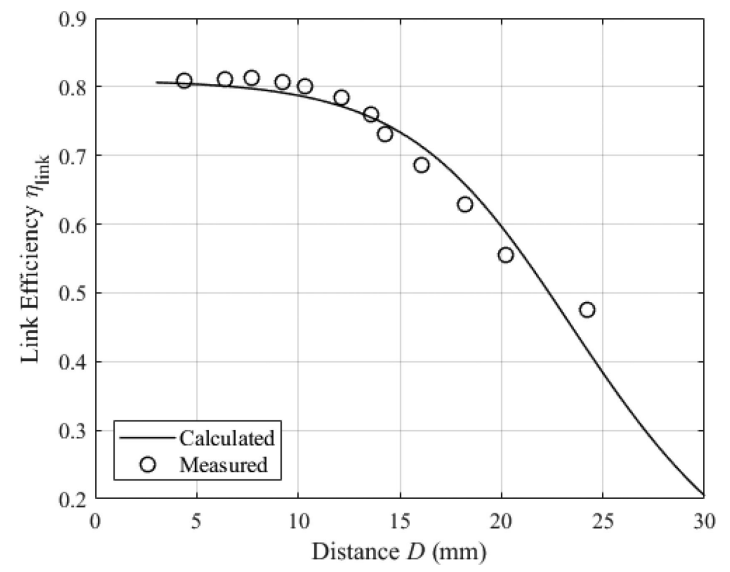

Fig. 25. Measured and calculated link efficiency for the constructed link, maintaining $40 \mathrm{~mW}$ at a $2 \mathrm{k} \Omega$ load.

and the $\alpha$ parameter [42]:

$$
\eta_{2}=\frac{Q_{2}}{\alpha+Q_{2}}
$$

where $\alpha=2 \pi f_{0} C_{2} R_{\text {out }}$, and $f_{0}$ is the resonant frequency of the Rx. The measured efficiency is shown in Fig. 25, and is in good agreement with the calculated prediction.

\section{CONCLUSION}

This paper has provided a detailed review of analytic methods for inductive link design; each method is discussed from first principles, to serve as a tutorial for newcomers to the subject. By focusing on methods that are both analytical and practical, biomedical power link designs can be realized without requiring FEM modelling and solving. While the primary focus of the paper has been on small biomedical power links, there is no reason the analyses cannot be applied to larger scale links, for example for industrial applications, as long as the coil shapes are sufficiently similar to solenoids or planar coils.

To further simplify the job of the link designer, the $\mathrm{CuCCo}$ software has been presented. This allows for automatic evaluation of the formulae throughout this paper, so that links can be designed, and their performance can be predicted, quickly and easily.

\section{APPENDIX A}

\section{Cumulative H-field Calculation}

A summary of the method for determining the cumulative $\mathrm{H}$-field experienced by a coil as a function of its geometry, given in [63] is presented below. First, each individual conductor (turn) is considered as being surrounded by immediately neighboring conductors, of which each will be put into either the pair group or the asymmetric group. The pair group consists of the conductors where a conductor on the left/right of the target conductor has a corresponding conductor on the right/left. The asymmetric group consists of the other conductors with no such corresponding conductor (see [63, Fig. 3]). The field on a conductor $m$ from left and right pair conductors $i$ and $j$ is defined as:

$$
H_{m, p a i r}(i, j)=-\frac{I_{0}}{2 \pi} \sqrt{\frac{p_{i m}^{2}+r_{0}^{2}}{\left(p_{i m}^{2}-r_{0}^{2}\right)^{2}}+\frac{p_{m j}^{2}+r_{0}^{2}}{\left(p_{m j}^{2}-r_{0}^{2}\right)^{2}}-\frac{2\left(p_{i m} p_{m j}-r_{0}^{2}\right)}{\left(p_{i m}^{2}-r_{0}^{2}\right)\left(p_{m j}^{2}-r_{0}^{2}\right)}},
$$

where $p_{i m}$ is the pitch between $i$ and $m$, and $p_{m j}$ is the pitch between $j$ and $m$. $H_{m, p a i r}$ must be calculated according to the following rules:

$$
H_{m, \text { pair }}= \begin{cases}0, & \text { for } m=1 \text { and } n \\ \sum_{i=1}^{m-1} H_{m, \text { pair }}(i, j), & \text { for } 1<m \leqslant n / 2 \\ \sum_{i=2 m-n}^{m-1} H_{m, \text { pair }}(i, j), & \text { for } n / 2<m<n .\end{cases}
$$

where $n$ is the number of conductors (turns). The field from the asymmetric group is calculated similarly; the field on a conductor $m$ from an asymmetrically adjacent conductor $k$ is defined as:

$$
H_{m, a s y}(k)=-\frac{I_{0}}{2 \pi} \frac{p_{m k}}{p_{m k}^{2}+r_{0}^{2}},
$$

where $p_{m k}$ is the distance between the conductors $m$ and $k$. $H_{m, a s y}$ must also be calculated in accordance with the following rules:

$$
H_{m, a s y}= \begin{cases}0, & \text { for } m=n / 2 \\ \sum_{k=2 m}^{n} H_{m, a s y}(k), & \text { for } 1 \leqslant m \leqslant n / 2 \\ \sum_{k=1}^{2 m-n-1} H_{m, a s y}(k), & \text { for } n / 2<m<n \\ \sum_{k=1}^{n-1} H_{m, a s y}(k), & \text { for } m=n .\end{cases}
$$

The total magnitude can then be calculated from the sum of the symmetric and asymmetric group fields:

$$
H_{m}=H_{m, p a i r}+H_{m, a s y}
$$

and the total field $H$ can be defined as the sum of all the $H_{m}$ values:

$$
H=\sum_{m=1}^{n} H_{m} .
$$

The result of (63) can be substituted back into (11) in order to predict proximity effect losses.

\section{APPENDIX B \\ USEFUL FORMULAE}

Table VI summarizes the formulae presented throughout the paper, as a point of reference for the designer. 
TABLE VI

COMmon Link Design Formulae: TABle of REFERENCE

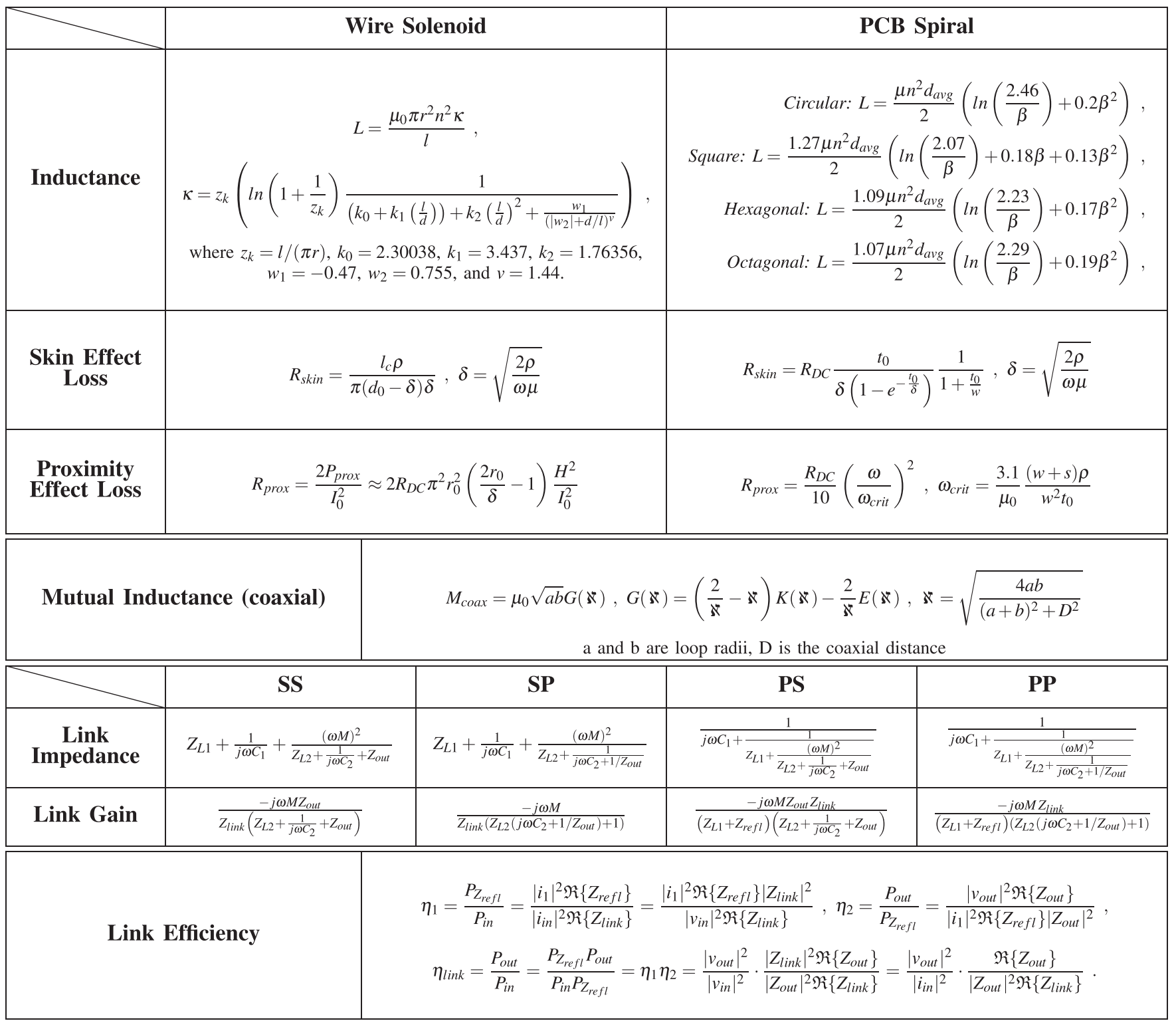

\section{REFERENCES}

[1] A. P. Sample, D. A. Meyer, and J. R. Smith, "Analysis, experimental results, and range adaptation of magnetically coupled resonators for wireless power transfer," IEEE Trans. Ind. Electron., vol. 58, no. 2, pp. 544-554, Feb. 2011.

[2] J. Shin et al., "Design and implementation of shaped magneticresonance-based wireless power transfer system for roadway-powered moving electric vehicles," IEEE Trans. Ind. Electron., vol. 61, no. 3, pp. 1179-1192, Mar. 2014.

[3] S. a. Mirbozorgi, H. Bahrami, M. Sawan, and B. Gosselin, "A smart multi-receiver power transmission system for long-term biological monitoring," in Proc. IEEE Biomed. Circuits Syst. Conf., 2014, pp. 412-415.

[4] Q. Li, Y. C. Liang, and S. Member, "An inductive power transfer system with a high-q resonant tank for mobile device charging," IEEE Trans. Power Electron., vol. 30, no. 11, pp. 6203-6212, Nov. 2015.

[5] M. J. Chabalko and A. P. Sample, "Three-dimensional charging via multimode resonant cavity enabled wireless power transfer," IEEE Trans. Power Electron., vol. 30, no. 11, pp. 6163-6173, Nov. 2015.

[6] H. Hoang, S. Lee, Y. Kim, Y. Choi, and F. Bien, "An adaptive technique to improve wireless power transfer for consumer electronics," IEEE Trans. Consumer Electron., vol. 58, no. 2, pp. 327-332, May 2012.
[7] N. Kyungmin, J. Heedon, S. K. Oruganti, and F. Bien, "An improved wireless power transfer system with adaptive technique for implantable biomedical devices," in Proc. IEEE MTT-S Int. Microw. Workshop Series RF Wireless Technol. Biomed. Healthcare Appl., 2013, pp. 1-3.

[8] M. W. Baker and R. Sarpeshkar, "Feedback analysis and design of RF power links for low-power bionic systems," IEEE Trans. Biomed. Circuits Syst., vol. 1, no. 1, pp. 28-38, Mar. 2007.

[9] P. Si, A. P. Hu, S. Malpas, and D. Budgett, "A frequency control method for regulating wireless power to implantable devices," IEEE Trans. Biomed. Circuits Syst., vol. 2, no. 1, pp. 22-29, Mar. 2008.

[10] R. Wu, W. Li, H. Luo, J. K. O. Sin, and C. P. Yue, "Design and characterization of wireless power links for brain machine interface applications," IEEE Trans. Power Electron., vol. 29, no. 10, pp. 5462-5471, Oct. 2014.

[11] G. Simard, M. Sawan, and D. Massicotte, "High-speed OQPSK and efficient power transfer through inductive link for biomedical implants," IEEE Trans. Biomed. Circuits Syst., vol. 4, no. 3, pp. 192-200, Jun. 2010.

[12] B. Lee, D. Ahn, and M. Ghovanloo, "Three-phase time-multiplexed planar power transmission to distributed implants," IEEE J. Emerg. Sel. Topics Power Electron., vol. 4, no. 1, pp. 263-272, Mar. 2016. 
[13] C.-y. Wu, X.-h. Qian, M.-s. Cheng, Y.-a. Liang, and W.-M. Chen, "A $13.56 \mathrm{MHz} 40 \mathrm{~mW}$ CMOS high-efficiency inductive link power supply utilizing on-chip delay-compensated voltage doubler rectifier and multiple LDOS for implantable medical devices," IEEE J. Solid-State Circuits, vol. 49, no. 11, pp. 2397-2407, Nov. 2014.

[14] W. Greatbatch and C. Holmes, "History of implantable devices," IEEE Eng. Med. Biol. Mag., vol. 10, no. 3, pp. 38-41, Sep. 1991.

[15] K. Bocan and E. Sejdić, "Adaptive transcutaneous power transfer to implantable devices: A state of the art review," Sensors, vol. 16, no. 3, 2016, Art. no. 393.

[16] K. Agarwal, R. Jegadeesan, Y.-X. Guo, and N. V. Thakor, "Wireless power transfer strategies for implantable bioelectronics," IEEE Rev. Biomed. Eng., vol. 10, pp. 136-161, Mar. 2017.

[17] J. Charthad, M. J. Weber, T. C. Chang, and A. Arbabian, "A mm-sized implantable medical device (IMD) with ultrasonic power transfer and a hybrid bi-directional data link," IEEE J. Solid-State Circuits, vol. 50, no. 8, pp. 1741-1753, Aug. 2015.

[18] M. D. Menz, O. Oralkan, P. T. Khuri-Yakub, and S. A. Baccus, "Precise neural stimulation in the retina using focused ultrasound," J. Neurosci., vol. 33, no. 10, pp. 4550-4560, Mar. 2013.

[19] R. Jegadeesan, K. Agarwal, Y.-X. Guo, S.-C. Yen, and N. V. Thakor, "Wireless power delivery to flexible subcutaneous implants using capacitive coupling," IEEE Trans. Microw. Theory Techn., vol. 65, no. 1, pp. 280-292, Jan. 2017.

[20] A. M. Sodagar and P. Amiri, "Capacitive coupling for power and data telemetry to implantable biomedical microsystems," in Proc. 4th Int. IEEE/EMBS Conf. Neural Eng., Apr. 2009, pp. 411-414.

[21] R. Erfani, F. Marefat, A. M. Sodagar, and P. Mohseni, "Modeling and experimental validation of a capacitive link for wireless power transfer to biomedical implants," IEEE Trans. Circuits Syst. II: Express Briefs, 2017, [Online]. Available: https://ieeexplore.ieee.org/document/8004478/

[22] W. H. Ko, J. Hyneck, and J. Homa, "Single frequency RF powered ECG telemetry system," IEEE Trans. Biomed. Eng., vol. BME-26, no. 2, pp. 105-109, Feb. 1979.

[23] M. Kiani and M. Ghovanloo, "An RFID-based closed-loop wireless power transmission system for biomedical applications," IEEE Trans. Circuits Syst. II, Express Briefs, vol. 57, no. 4, pp. 260-264, Apr. 2010.

[24] U. Çilingirolu and I. Sercan, "A zero-voltage switching technique for minimizing the current-source power of implanted stimulators," IEEE Trans. Biomed. Circuits Syst., vol. 7, no. 4, pp. 469-479, Aug. 2013.

[25] D. Jiang, D. Cirmirakis, M. Schormans, T. A. Perkins, N. Donaldson, and A. Demosthenous, "An integrated passive phase-shift keying modulator for biomedical implants with power telemetry over a single inductive link," IEEE Trans. Biomed. Circuits Syst., vol. 11, no. 1, pp. 64-77, Feb. 2017.

[26] O. Knecht, R. Bosshard, and J. W. Kolar, "High-efficiency transcutaneous energy transfer for implantable mechanical heart support systems," IEEE Trans. Power Electron., vol. 30, no. 11, pp. 6221-6236, Nov. 2015.

[27] S. A. Mirbozorgi, P. Yeon, and M. Ghovanloo, "Robust wireless power transmission to mm-sized free-floating distributed implants," IEEE Trans. Biomed. Circuits Syst., vol. 11, no. 3, pp. 692-702, Jun. 2017.

[28] Y. Wang, D. Ye, L. Lyu, Y. Xiang, H. Min, and C.-J. R. Shi, "A $13.56 \mathrm{mhz}$ wireless power and data transfer receiver achieving $75.4 \%$ effectivepower-conversion efficiency with $0.1 \%$ ask modulation depth and $9.2 \mathrm{mw}$ output power," in Proc. IEEE Int. Solid - State Circuits Conf., 2018, pp. $142-144$

[29] F. E. Terman, Radio Engineering. New York, NY, USA: McGraw-Hill, 1937.

[30] F. E. Terman, Radio Engineers' Handbook. New York, NY, USA: McGraw-Hill, 1943.

[31] U.-M. Jow and M. Ghovanloo, "Modeling and optimization of printed spiral coils in air, saline, and muscle tissue environments," IEEE Trans. Biomed. Circuits Syst., vol. 3, no. 5, pp. 339-347, Oct. 2009.

[32] W. H. Ko, S. P. Liang, and C. D. F. Fung, "Design of radio-frequency powered coils for implant instruments," Med. Biol. Eng. Comput., vol. 15, no. 6, pp. 634-640, 1977.

[33] N. d. N. Donaldson and T. A. Perkins, "Analysis of resonant coupled coils in the design of radio frequency transcutaneous links," Med. Biol. Eng. Comput., vol. 21, no. Sep., pp. 612-627, 1983.

[34] K. Fotopoulou and B. W. Flynn, "Wireless power transfer in loosely coupled links: Coil misalignment model," IEEE Trans. Magn., vol. 47, no. 2, pp. 416-430, Feb. 2011.

[35] B. X. Nguyen et al., "An efficiency optimization scheme for bidirectional inductive power transfer systems," IEEE Trans. Power Electron., vol. 30, no. 11 , pp. 6310-6319, Nov. 2015.
[36] M. E. Halpern and D. C. Ng, "Optimal tuning of inductive wireless power links: Limits of performance," IEEE Trans. Circuits Syst. I, Reg. Papers, vol. 62, no. 3, pp. 725-732, Mar. 2015.

[37] W.-Q. Niu, J.-X. Chu, W. Gu, and A.-D. Shen, "Exact analysis of frequency splitting phenomena of contactless power transfer systems," IEEE Trans. Circuits Syst. I, Reg. Papers, vol. 60, no. 6, pp. 1670-1677, Jun. 2013

[38] W. Zhong, C. K. Lee, and S. Y. Ron Hui, "General analysis on the use of Tesla's resonators in domino forms for wireless power transfer," IEEE Trans. Ind. Electron., vol. 60, no. 1, pp. 261-270, Jan. 2013.

[39] Y. Ahn, I. Jeon, and J. Roh, "A multiphase buck converter with a rotating phase-shedding scheme for efficient light-load control," IEEE J. SolidState Circuits, vol. 49, no. 11, pp. 2673-2683, Nov. 2014.

[40] Z. Dang, Y. Cao, and J. A. Abu Qahouq, "Reconfigurable magnetic resonance-coupled wireless power transfer system," IEEE Trans. Power Electron., vol. 30, no. 11, pp. 6057-6069, Nov. 2015.

[41] E. Moradi, T. Bjorninen, L. Sydanheimo, L. Ukkonen, and J. M. Rabaey, "Analysis of wireless powering of mm-size neural recording tags in rfidinspired wireless brain-machine interface systems," in Proc. IEEE Int. Conf. RFID, Apr. 2013, pp. 8-15.

[42] K. Van Schuylenbergh and R. Puers, Eds., Inductive Powering. Dordrecht, The Netherlands: Springer, 2009. [Online]. Available: http://link.springer.com/10.1007/978-90-481-2412-1

[43] CuCCo: The coupled coil configurator, 2018. [Online]. Available: https://github.com/schormans/CuCCo

[44] M. Kiani, U. M. Jow, and M. Ghovanloo, "Design and optimization of a 3-coil inductive link for efficient wireless power transmission," IEEE Trans. Biomed. Circuits Syst., vol. 5, no. 6, pp. 579-591, Dec. 2011.

[45] N. Hoang and I. A. Johnson, "Splitting frequency diversity in wireless power transmission," IEEE Trans. Power Electron., vol. 30, no. 11, pp. 6088-6096, Nov. 2015.

[46] H. A. Wheeler, "Simple inductance formulas for radio coils," Proc. Inst. Radio Eng., vol. 16, no. 10, pp. 1398-1400, 1928.

[47] F. W. Grover, Inductance Calculations. New York, NY, USA: Dover, 1962.

[48] P. Silvester, Modern Electromagnetic Fields. Englewood Cliffs, NJ, USA: Prentice-Hall, 1968. [Online]. Available: https://archive.org/ details/ModernElectromagneticFields

[49] W. G. Hurley et al., "A unified approach to the calculation of self- and mutual-inductance for coaxial coils in air," IEEE Trans. Power Electron., vol. 30, no. 11, pp. 6155-6162, Nov. 2015.

[50] K. A. Wirgau, "Inductance calculation of an air-core disk winding," IEEE Trans. Power App. Syst., vol. PAS-95, no. 1, pp. 394-400, Jan. 1976.

[51] D. W. Knight, "An introduction to the art of solenoid inductance calculation," 2016. [Online]. Available: http://g3ynh.info/zdocs/magnetics/ Solenoids.pdf

[52] S. S. Mohan, M. D. M. Hershenson, S. P. Boyd, and T. H. Lee, "Simple accurate expressions for planar spiral inductances," IEEE J. Solid-State Circuits, vol. 34, no. 10, pp. 1419-1420, Oct. 1999.

[53] H. B. Dwight, "Skin effect in tubular and flat conductors," Trans. Am. Inst. Electr. Eng., vol. XXXVII, no. 2, pp. 1379-1403, Jul. 1918.

[54] J. Cockcroft, "Skin effect in rectangular conductors at high frequencies," Proc. Royal Soc. London. Ser. A, Containing Papers Math. Phys. Character, vol. 122, no. 790, pp. 533-542, 1928.

[55] U.-m. Jow and M. Ghovanloo, "Design and optimization of printed spiral coils for efficient inductive power transmission," in Proc. 14th IEEE Int. Conf. Electron., Circuits Syst., 2007, pp. 70-73.

[56] J. Lammeraner and M. Stafl, Eddy Currents. London, U.K.: Iliffe Books Ltd., 1966.

[57] P. Meyer, P. Germano, and Y. Perriard, "FEM modeling of skin and proximity effects for coreless transformers," in Proc. Int. Conf. Elect. Mach. Syst., 2012, pp. 1-6.

[58] I. Lope, C. Carretero, J. Acero, R. Alonso, and J. M. Burdío, "AC power losses model for planar windings with rectangular cross-sectional conductors," IEEE Trans. Power Electron., vol. 29, no. 1, pp. 23-28, Jan. 2014.

[59] G. Klaric Felic, D. Ng, and E. Skafidas, "Investigation of frequencydependent effects in inductive coils for implantable electronics," IEEE Trans. Magn., vol. 49, no. 4, pp. 1353-1360, Apr. 2013.

[60] R. C. Fernandes and A. A. de Oliveira, "Iterative design method of weakly coupled magnetic elements for inductive power transfer," in Proc. Brazilian Power Electron. Conf., Oct. 2013, pp. 1088-1094.

[61] S.-H. Lee and R. D. Lorenz, "Development and validation of model for 95\%-efficiency 220 -w wireless power transfer over a 30 -cm air gap," IEEE Trans. Ind. Appl., vol. 47, no. 6, pp. 2495-2504, Nov. 2011. 
[62] Z. Pantic and S. Lukic, "Computationally-efficient, generalized expressions for the proximity-effect in multi-layer, multi-turn tubular coils for wireless power transfer systems," IEEE Trans. Magn., vol. 49, no. 11, pp. 5404-5416, Nov. 2013.

[63] J. Kim and Y. Park, "Approximate closed-form formula for calculating ohmic resistance in coils of parallel round wires with unequal pitches," IEEE Trans. Ind. Electron., vol. 62, no. 6, pp. 3482-3489, Jun. 2015

[64] W. B. Kuhn and N. M. Ibrahim, "Analysis of current crowding effects in multiturn spiral inductors," IEEE Trans. Microw. Theory Techn., vol. 49, no. 1, pp. 31-38, Jan. 2001.

[65] H. Wheeler, "Formulas for the skin effect," Proc. IRE, vol. 30, no. 9, pp. 412-424, Sep. 1942.

[66] B. H. Choi, E. S. Lee, J. Huh, and C. T. Rim, "Lumped impedance transformers for compact and robust coupled magnetic resonance systems," IEEE Trans. Power Electron., vol. 30, no. 11, pp. 6046-6056, Nov. 2015.

[67] J. T. Boys, G. Covic, and A. W. Green, "Stability and control of inductively coupled power transfer systems," IEE Proc.-Electr. Power Appl., vol. 147, no. 1, pp. 37-43, 2000.

[68] Q. Ke, W. Luo, G. Yan, and K. Yang, "Analytical model and optimized design of power transmitting coil for inductive coupled endoscope robot," IEEE Trans. Biomed. Eng., vol. 63, no. 4, pp. 694-706, Apr. 2016.

[69] G. A. Kendir et al., "An optimal design methodology for inductive power link with class-e amplifier," IEEE Trans. Circuits Syst. I, Reg. Papers, vol. 52, no. 5, pp. 857-866, May 2005.

[70] M. Bartoli, N. Noferi, A. Reatti, and M. K. Kazimierczuk, "Modeling litz-wire winding losses in high-frequency power inductors," in Proc. IEEE Annu. Power Electron. Spec. Conf., 1996, pp. 1690-1696.

[71] C. Carretero, J. Acero, and R. Alonso, "TM-TE decomposition of power losses in multi-stranded litz-wires used in electronic devices," Progr. Electromagn. Res., vol. 123, pp. 83-103, 2012.

[72] M. Etemadrezaei and S. Lukic, "Coated-strand litz wire for multi-MHz frequency applications," IEEE Trans. Magn., vol. 52, no. 8, Aug. 2016, Art. no. 6301511.

[73] J. Acero, P. J. Hernández, J. M. Burdío, R. Alonso, and L. A. Barragán, "Simple resistance calculation in litz-wire planar windings for induction cooking appliances," IEEE Trans. Magn., vol. 41, no. 4, pp. 1280-1288, Apr. 2005.

[74] A. J. Palermo, "Distributed capacity of single-layer," Proc. Inst. Radio Eng., vol. 22, no. 7, pp. 897-905, 1934.

[75] Z. Jiang, P. S. Excell, and Z. M. Hejazi, "Calculation of distributed capacitances of spiral resonators," IEEE Trans. Microw. Theory Techn., vol. 45, no. 1, pp. 139-142, Jan. 1997.

[76] J. Biela and J. W. Kolar, "Using transformer parasitics for resonant converters-A review of the calculation of the stray capacitance of transformers," IEEE Trans. Ind. Appl., vol. 44, no. 1, pp. 223-233, Jan./Feb. 2008.

[77] M. B. Shadmand and R. S. Balog, "Determination of parasitic parameters in a high frequency magnetic to improve the manufacturability, performance, and efficiency of a pv inverter," in Proc. Conf. Record IEEE Photovolt. Spec. Conf., 2012, pp. 1368-1372.

[78] Payne Alan, "Self-resonance in coils and the self-capacitance myth," vol. 6, pp. 1-11, 2014. [Online]. Available: http://g3rbj.co.uk/wpcontent/uploads/2014/07/Self-Resonance-in-Coils.pdf

[79] J. Groszkowski, Frequency of Self-Oscillations. Amsterdam, The Netherlands: Elsevier, 1964.

[80] M. Soma, D. C. Galbraith, and R. L. White, "Radio-frequency coils in implantable devices: Misalignment analysis and design procedure," IEEE Trans. Biomed. Eng., vol. BME-34, no. 4, pp. 276-282, Apr. 1987.

[81] J. S. Ho et al., "Wireless power transfer to deep-tissue microimplants," Proc. Nat. Acad. Sci., vol. 111, no. 22, pp. 7974-7979, Jun. 2014.

[82] F. C. Flack, E. D. James, and D. M. Schlapp, "Mutual inductance of air-cored coils: Effect on design of radio-frequency coupled implants," Med. Biol. Eng., vol. 9, no. 2, pp. 79-85, Mar. 1971.

[83] C. Zierhofer and E. Hochmair, "Geometric approach for coupling enhancement of magnetically coupled coils," IEEE Trans. Biomed. Eng., vol. 43, no. 7, pp. 708-714, Jul. 1996.

[84] T. Ohira, "The kq product as viewed by an analog circuit engineer," IEEE Circuits Syst. Mag., vol. 17, no. 1, pp. 27-32, First Quarter 2017.

[85] K. Hata, T. Imura, and Y. Hori, "Simplified measuring method of kq product for wireless power transfer via magnetic resonance coupling based on input impedance measurement," in Proc. 43rd Annu. Conf. IEEE Ind. Electron. Soc., Oct. 2017, pp. 6974-6979.

[86] T. Ujihara, Q. T. Duong, and M. Okada, "Kq-product analysis of inductive power transfer system with two transmitters and two receivers," in Proc. Wireless Power Transfer Conf., 2017, pp. 2-5.
[87] A. Barchanski, "Simulations of the low-frequency electromagnetic fields in the human body," Ph.D. dissertation, Technischen Universität Darmstadt, Darmstadt, Germany, 2007.

[88] X. P. Ripoll, "Electrical properties of human tissues applied to wearable antenna design," Ph.D. dissertation, Univ. Colorado Boulder, Boulder, CO, USA, 2012.

[89] A. Trigui, S. Hached, F. Mounaim, and A. C. Ammari, "Inductive power transfer system with self-calibrated primary resonant frequency," IEEE Trans. Power Electron., vol. 30, no. 11, pp. 6078-6087, Nov. 2015.

[90] S. J. Thomas and M. S. Reynolds, "A 96 mbit/sec, 15. 5 pj / bit 16 QAM modulator for UHF backscatter communication," in Proc. IEEE Int. Conf. RFID, 2012, pp. 185-190.

[91] Y. Zhang and Z. Zhao, "Frequency splitting analysis of two-coil resonant wireless power transfer," IEEE Antennas Wireless Propag. Lett., vol. 13, pp. 400-402, Feb. 2014.

[92] Y. Zhang, Z. Zhao, and K. Chen, "Frequency-splitting analysis of fourcoil resonant wireless power transfer," IEEE Trans. Ind. Appl., vol. 50, no. 4, pp. 2436-2445, Jul. 2014.

[93] Y.-1. Lyu et al., "A method of using nonidentical resonant coils for frequency splitting elimination in wireless power transfer," IEEE Trans. Power Electron., vol. 30, no. 11, pp. 6097-6107, Nov. 2015.

[94] M. Schormans, V. Valente, and A. Demosthenous, "Efficiency optimization of class-d biomedical inductive wireless power transfer systems by means of frequency adjustment," Proc. Annu. Int. Conf. IEEE Eng. Med. Biol. Soc., 2015, pp. 5473-5476.

[95] M. Schormans, V. Valente, and A. Demosthenous, "Frequency splitting analysis and compensation method for inductive wireless powering of implantable biosensors," Sensors, vol. 16, no. 8, 2016, Art. no. E1229.

[96] F.-G. Zeng, S. Rebscher, W. Harrison, X. Sun, and H. Feng, "Cochlear implants: System design, integration, and evaluation," IEEE Rev. Biomed. Eng., vol. 1, pp. 115-142, Nov. 2008.

[97] G. Wang, W. Liu, M. Sivaprakasam, and G. A. Kendir, "Design and analysis of an adaptive transcutaneous power telemetry for biomedical implants," IEEE Trans. Circuits Syst. I, Reg. Papers, vol. 52, no. 10, pp. 2109-2117, Oct. 2005.

[98] R. Carta and R. Puers, "Wireless power and data transmission for robotic capsule endoscopes," in Proc. 18th IEEE Symp. Commun. Veh. Technol. Benelux, 2011, pp. 1-6.

[99] M. R. Basar, M. Yazed, J. Cho, and F. Ibrahim, "A wireless power transmission system for robotic capsule endoscopy: Design and optimization," in Proc. IEEE MTT-S Int. Microw. Workshop Series RF Wireless Technol. Biomed. Healthcare Appl., 2014, pp. 2-4.

[100] Y. Yang, X. Xie, G. Li, Y. Huang, and Z. Wang, "A combined transmitting coil design for high efficiency WPT of endoscopic capsule," in Proc. Int. Symp. Circuits Syst., 2015, pp. 97-100.

[101] K. Sasaki, S. Sugiura, and H. Iizuka, "Distance adaptation method for magnetic resonance coupling between variable capacitor-loaded parallelwire coils," IEEE Trans. Microw. Theory Techn., vol. 62, no. 4, pp. 892900, Apr. 2014

[102] M. Kiani and M. Ghovanloo, "A closed loop wireless power transmission system using a commercial RFID transceiver for biomedical applications," in Proc. Annu. Int. Conf. IEEE Eng. Med. Biol. Soc., 2009, pp. 3841-3844

[103] C. Zheng, R. Chen, and J.-S. Lai, "Design considerations to reduce gap variation and misalignment effects for inductive power transfer system," IEEE Trans. Power Electron., vol. 30, no. 11, pp. 1384-1390, Nov. 2015.

[104] R. Jegadeesan and Y.-X. Guo, "Topology selection and efficiency improvement of inductive power links," IEEE Trans. Antennas Propag., vol. 60, no. 10, pp. 4846-4854, Oct. 2012.

[105] P. Aqueveque, M. Saez, J. E. Rodriguez, and E. Pino, "An inductive-link with a regulated secondary voltage based on frequency adjustment," in Proc. Annu. Int. Conf. IEEE Eng. Med. Biol. Soc., Aug. 2012, pp. 16711674.

[106] M. Catrysse, B. Hermans, and R. Puers, "An inductive power system with integrated bi-directional data-transmission," Sens. Actuators A: Phys., vol. 115, nos. 2/3, pp. 221-229, Sep. 2004.

[107] V. Valente, C. Eder, N. Donaldson, and A. Demosthenous, "A high-power CMOS class-d amplifier for inductive-link medical transmitters," IEEE Trans. Power Electron., vol. 30, no. 8, pp. 4477-4488, Aug. 2015

[108] R. Bhattacharyya and M. E. McCormick, "Analysis and tracking of optimal load inwireless power transfer systems," IEEE Trans. Power Electron., vol. 30, no. 7, pp. 3952-3963, Jul. 2015

[109] L. Cheng, W. H. Ki, Y. Lu, and T. S. Yim, "Adaptive on/off delaycompensated active rectifiers for wireless power transfer systems," IEEE J. Solid-State Circuits, vol. 51, no. 3, pp. 712-723, Mar. 2016. 
[110] Y. Lu and W. H. Ki, "A 13.56 MHz CMOS active rectifier with switchedoffset and compensated biasing for biomedical wireless power transfer systems," IEEE Trans. Biomed. Circuits Syst., vol. 8, no. 3, pp. 334-344, Jun. 2014.

[111] E. Waffenschmidt, "Dynamic resonant matching method for a wireless power transmission receiver," IEEE Trans. Power Electron., vol. 30, no. 11 , pp. 6070-6077, Nov. 2015.

[112] "GNU general public license," Free Software Foundation. 2007. [Online]. Available: http://www.gnu.org/licenses/gpl.html

[113] O. Ozturan, A. Yenigun, E. Senturk, O. F. Calim, F. Aksoy, and S. B. Eren, "Temporal scalp thickness, body mass index, and suprafascial placement of receiver coil of the cochlear implant," J. Craniofacial Surg., vol. 28, no. 8, pp. e781-e785, Nov. 2017.

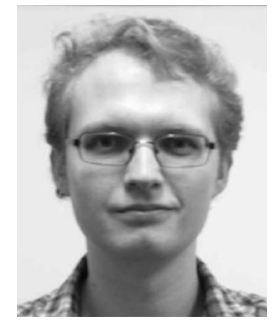

Matthew Schormans (S'14) received the M.Eng. degree in electronic and electrical engineering from the University College London, London, U.K., in 2014, where he is also currently working toward the Ph.D. degree in electronic and electrical engineering, with a focus on short-range wireless power and data telemetry for implantable medical devices.

He has authored several conference and journal publications on biomedical systems employing inductive links and techniques for employing inductive links for both power and data transfer in a biomedical context.

His research is concerned primarily with biomedical wireless power telemetry systems, but his interests also include inductive link modelling, oscillators, and more general power converter and mixed signal design.

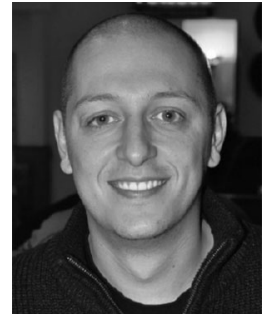

Virgilio Valente (S'09-M'11) was born in Milan, Italy, in 1979. He received the B.Sc. degree (first class honrs.) in electronic engineering in 2004 from the University of York, York, U.K., the M.Sc. degree in biomedical engineering in 2006 from Aalborg University, Aalborg, Denmark, and the Ph.D. degree in electronic and electrical engineering from University College London (UCL), London, U.K., in 2011. From 2011 to 2017, he was a Research Associate with the Analog and Biomedical Electronics Group, UCL, during which, in 2015, he also joined Tetrivis Ltd., U.K., as an IC Design Engineer. In 2017, he was a Visiting Scholar with the Nano Lab, Tufts University, Medford, MA, USA. He is currently an Assistant Professor (Research) in bioelectronics, within the Department of Microelectronics, Delft University of Technology, Delft, The Netherlands.

His research interests focus on the development of analog and mixed-mode CMOS integrated circuits for a range of applications, including BioCMOS, lab-on-CMOS and organ-on-chip platforms, implantable and injectable CMOS biosensors, CMOS-microfluidic devices and smart wireless telemetry systems.

Dr. Valente is a member of the Biomedical Circuits and Systems Technical Committee of the IEEE Circuits and Systems Society and the recipient of the 2017 EPSRC IRC i-sense Mobility Fellowship.

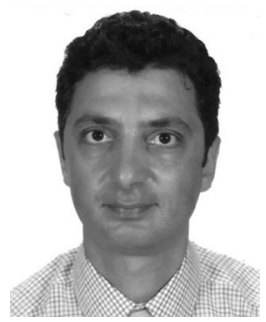

Andreas Demosthenous (S'94-M'99-SM'05F'18) received the B.Eng. degree in electrical and electronic engineering from the University of Leicester, Leicester, U.K., the M.Sc. degree in telecommunications technology from Aston University, Birmingham, U.K., and the Ph.D. degree in electronic and electrical engineering from the University College London (UCL), London, U.K., in 1992, 1994, and 1998, respectively. $\mathrm{He}$ is a Professor with the UCL Department of Electronic and Electrical Engineering and leads the Analog and Biomedical Electronics Group. His research interests include analog and mixed-signal integrated circuits for biomedical, sensor, and signal processing applications. He has made outstanding contributions to improving safety and performance in integrated circuit design for active medical devices, such as spinal cord and brain stimulators. He has authored more than 300 articles in journals and international conference proceedings, several book chapters, and holds several patents.

$\mathrm{He}$ is currently the Editor-in-Chief of the IEEE TRANSACTIONS ON Circuits and Systems I: Regular PaPers, an Associate Editor of the IEEE TRANSACTIONS ON BIOMEDICAL CIRCUITS AND Systems, and serves on the International Advisory Board of Physiological Measurement. He is a member of the Technical Programme Committee of several IEEE conferences, including the European Solid-State Circuits Conference and the International Symposium on Circuits and Systems.

Dr. Demosthenous is a Fellow of the Institution of Engineering and Technology and a Chartered Engineer. 